\title{
Genetic architecture of floral traits in bee- and hummingbird- pollinated sister species of Aquilegia (columbine)
}

\author{
Molly B. Edwards ${ }^{1}$, Gary P. T. Choi ${ }^{2}$, Nathan J. Derieg, Ya Min ${ }^{1}$, Angie C. Diana ${ }^{1}$, Scott A.
} Hodges $^{3}$, L. Mahadevan ${ }^{1,4,5}$, Elena M. Kramer ${ }^{1}$, Evangeline S. Ballerini ${ }^{3,6 *}$

1. Dept. of Organismic \& Evolutionary Biology, Harvard University; 2. Dept. of Mathematics, Massachusetts Institute of Technology; 3. Dept. of Ecology, Evolutionary, and Marine Biology, University of California Santa Barbara; 4. School of Engineering \& Applied Sciences, Harvard University; 5. Dept. of Physics, Harvard University; 6. Dept. of Biological Sciences, California State University Sacramento.

*Corresponding Author, ballerini@csus.edu

Key words: Aquilegia, floral trait, pollination syndrome, flower morphology, genetic architecture, anthocyanin pigmentation

\section{Abstract}

2 Interactions with animal pollinators have helped shape the stunning diversity of flower

3 morphologies across the angiosperms. A common evolutionary consequence of these interactions

4 is that some flowers have converged on suites of traits, or pollination syndromes, that attract and reward specific pollinator groups. Determining the genetic basis of these floral pollination syndromes can help us understand the processes that contributed to the diversification of the angiosperms. Here, we characterize the genetic architecture of a bee-to-hummingbird pollination shift in Aquilegia (columbine) using QTL mapping of 17 floral traits encompassing color, nectar

9 composition, and organ morphology. In this system, we find that the genetic architectures underlying differences in floral color are quite complex, and we identify several likely candidate

11 genes involved in anthocyanin and carotenoid floral pigmentation. Most morphological and

12 nectar traits also have complex genetic underpinnings; however, one of the key floral

13 morphological phenotypes, nectar spur curvature, is shaped by a single locus of large effect. 


\section{Introduction}

Pollinator interactions are a major force in shaping the evolutionary trajectory of

18 (Grant 1949; Fenster et al. 2004). For example, the bee pollination syndrome is typified by

21 provide abundant dilute nectar; and flowers pollinated by hawkmoths have even longer corolla

22 tubes, and produce heady fragrances but little floral pigment to aid in nocturnal detection.

23 Distantly related species have converged on these pollination syndromes: for instance, species in

24 genera as disparate as Costus (Costaceae), Phygelius (Scrophulariaceae), and Aquilegia

25 (Ranunculaceae) all exhibit the suite of floral traits typical of hummingbird-pollination (Cronk

26 and Ojeda 2008). Closely-related groups have also experienced multiple, independent shifts to

27 the same pollination syndrome; Penstemon is an extreme example, with an estimated 10-21

28 independent transitions from bee to hummingbird pollination having occurred within the genus

29 during a recent rapid radiation (Wilson et al. 2007). Indeed, pollinator specialization is associated

30 with species diversity and rapid speciation events in the angiosperms (Armbruster and Muchhala

31 2009).

The changes in individual floral traits that accompany these pollination syndrome shifts are profound, and must also be coordinated so that the whole flower can reach a new adaptive

34 optimum for pollinator attraction, reward, and pollen transfer. Illuminating the genetic

35 underpinnings of floral trait evolution during pollinator-driven rapid radiation can give us insight 
into some of the processes that generated the staggering diversity of the angiosperms. A common hypothesis is that biochemical traits such as flower color and nectar composition are controlled by few loci of large effect, such that a change in a single gene can lead to dramatic alteration of phenotype (Rockman 2012; Sheehan et al. 2012). On the other hand, morphological traits such as petal shape and stigma length are generally thought to be governed by many loci of minor effect. Quantitative trait locus (QTL) mapping studies in systems with contrasting pollination syndromes suggest these patterns hold true to some extent, but exceptions are common (Galliot et al. 2006; Hermann and Kuhlemeier 2011). As sequencing technology becomes increasingly accessible and more species are studied, it is unclear how universal this dichotomy between biochemical and morphological traits will remain.

The genus Aquilegia (columbine, Ranunculaceae) is an ideal study system for examining the genetic basis of pollination syndrome evolution. It is characterized by a key innovation, the petal nectar spur, that allows for pollinator specialization and facilitated the rapid radiation of the genus (Hodges and Arnold 1995). Aquilegia originated approximately 6.9 million years ago in eastern Asia; while there is considerable species diversity in Eurasian taxa, the vast majority

51 exhibit the bee-pollination syndrome, characterized by blue-purple flowers, and petals with long blades for landing as well as short curved nectar spurs (Hodges et al. 2004; Fior et al. 2013).

53 When the genus migrated to North America c. 4.8 million years ago, it experienced rapid 54 diversification to adapt to new pollinators (Whittall and Hodges 2007; Fior et al. 2013). Early in 55 this radiation, there were two independent transitions to hummingbird-pollination, characterized by elongation and straightening of the petal nectar spur, reduction of the petal blade length,

57 exertion of the reproductive organs, and a shift from blue to red floral pigmentation.

58 Subsequently, there were five shifts from hummingbird to hawkmoth pollination, involving 
59 further elongation of the nectar spur (up to $16 \mathrm{~cm}$ in the case of A. longissima), loss of

60 anthocyanin pigments, and acquisition of floral scent. There are hawkmoths in Eurasia, but

61 Aquilegia only experienced this pollination syndrome evolution once it reached North America,

62 where there are both hummingbirds and hawkmoths. Whittall and Hodges (2007) suggested that

63 hummingbirds, with tongue lengths in between those of bees and moths, served as a necessary

64 intermediate in the evolution of spur length and accompanying floral trait shifts. Therefore,

65 characterizing this initial transition from bee- to hummingbird-pollination is essential for

66 understanding the diversification of the genus as a whole.

In this study, we use QTL mapping of floral traits in the only pair of Aquilegia sister species with these contrasting pollination syndromes to begin to elucidate the genetic

69 underpinnings of the genus's rapid radiation, and to assess whether any patterns are in line with

70 QTL studies of pollination syndromes in other systems. A. brevistyla exhibits the bee syndrome,

71 and is found in alpine regions in the northwestern United States and Canada (Fig. 1A; Roe 1992).

72 Its sister species, A. canadensis, is hummingbird-pollinated and has a more cosmopolitan

73 distribution across much of North America (Fig. 1A; Herlihy and Eckert 2005). They represent

74 the only pair of sister species in the genus with bee- and hummingbird-pollination, and are

75 estimated to have diverged less than 3 million years ago (Whittall and Hodges 2007; Fior et al.

76 2013). This study is the first genetic characterization of a bee-to-hummingbird shift in Aquilegia,

77 and is unique in the breadth and depth of traits examined, with 17 floral traits spanning color,

78 nectar composition, and organ morphology. 


\section{Materials and Methods}

\section{$83 \quad$ Genetic cross and growth conditions}

A. brevistyla seeds were collected from a wild population near Tucker Lake in Alberta,

85 Canada, and A. canadensis seeds from a wild population in Ithaca, New York. The parent plants

86 were grown from this seed in the greenhouse facilities at the University of California Santa

87 Barbara. An A. brevistyla paternal plant and an A. canadensis maternal plant were crossed to

88 produce the F1 generation (Fig. 1A). Five F1 plants were self-pollinated to produce the F2

89 population. Seeds were shipped to Harvard University, where approximately 2000 seeds were

90 sown in plug trays containing Fafard $3 \mathrm{~B}$ Soil and stratified at $4^{\circ} \mathrm{C}$ for 4 weeks. They were then

91 moved to growth chambers programmed to 16 hour days at $18^{\circ} \mathrm{C}$, and $13^{\circ} \mathrm{C}$ nights. 366 plants

92 germinated and were transferred to 6-inch pots and again to gallon pots as they matured. When

93 they had produced 11 leaves, they were vernalized at $4^{\circ} \mathrm{C}$ for 8 weeks to promote flowering. We

94 staggered stratification, transplanting, and vernalization in order to make phenotyping more

95 manageable. The first batch of plants (143 total, the offspring of two of the five F1s) were grown

96 post-vernalization in the greenhouse facilities at the Arnold Arboretum of Harvard University.

97 The second batch of plants (223 total, the offspring of the other three F1s) were grown post-

98 vernalization in the greenhouse facilities at Harvard University's main Cambridge campus. Both

99 batches experienced the same light intensity and day length; the Arboretum plants experienced

100 stable temperatures of $18^{\circ} \mathrm{C}$ during the day and $13^{\circ} \mathrm{C}$ at night, but the Harvard campus

101 greenhouse experienced warmer temperatures due to limited cooling ability. In total, 352 plants

102 reached the flowering stage. 
Genotyping

A multiplexed shotgun sequencing approach was used to genotype the F2s (Filiault et al.

107 2018). DNA was extracted from young leaf tissue collected from each F0 parent (flash frozen)

108 and each F2 (desiccated using silica gel) using Qiagen DNEasy reagents and Magattract beads

109 (Qiagen, Inc.). Sequencing libraries for the two F0 parents were prepared using the NEBNext

110 Ultra II kit (NEB) and sequenced to $\sim 40 \mathrm{x}$ coverage as $150 \mathrm{bp}$ reads on an Illumina MiSeq at the

111 Biological Nanostructures Lab in the California NanoSystem Institute at UC Santa Barbara. For

112 the F2s, DNA was quantified using a Qubit 2.0 fluorometer (ThermoFisher Scientific), and

113 100ng of each sample was used to prepare sequencing libraries with the iGenomX RIPTIDE kit.

114 Libraries were pooled and sequenced as 150bp paired-end reads in one lane of an Illumina

115 NovaSeq 6000 by the DNA Technologies \& Expression Analysis Core at the UC Davis Genome

116 Center to achieve $\sim 1-2 \mathrm{x}$ coverage for each F2.

117 All sequence reads were aligned to the $A$. coerulea 'Goldsmith' v3.1 reference genome

118 (https://phytozome.jgi.doe.gov) using the Burrows-Wheeler aligner (Li and Durbin 2009) as in

119 Filiault et al. (2018). Variable sites in the parents were identified using SAMtools 0.1.19 (Li et al.

120 2009) and custom scripts were used to identify the positions and genotypes at which the parents

121 were homozygous for different alleles. These sites were used to assign reads in the F2s as having

122 either $A$. canadensis or $A$. brevistyla ancestry. The genome was broken up into windows of either

$1230.5 \mathrm{Mb}$ or $1 \mathrm{Mb}$, depending on recombination rate as determined in prior crosses, and the

124 frequency of reads with ancestry for each F0 parent was used to determine the genotype of the

125 bin (see Filiault et al. 2018, Ballerini et al. 2020 for details). These bins and genotypes were used

126 as markers to construct a genetic map and conduct QTL mapping (see below). 
128

130

\section{Phenotyping}

Seventeen traits were phenotyped in the F2s and parents, spanning floral color and nectar composition, as well as sepal, petal, and pistil morphology (Table S1). Phenotypes were collected from nine flowers per plant, divided into three sets of three. The first set was used to phenotype all of the morphological traits, and the flower color found in the sepals and petal nectar spurs. The second set was used to phenotype the petal blade color. The third set was used to phenotype the nectar traits. All flowers were phenotyped at anthesis after approximately half of the anthers had dehisced.

Where possible, the three flowers collected for the first set were the terminal flower and the first two lateral flowers on the primary inflorescence. Sepals were removed and taped to a piece of black paper with their adaxial side up using clear packing tape; petals were removed, folded longitudinally, and also taped to the paper; and the inner floral whorls (stamens, staminodes, pistils) were left intact. These organs were scanned with a ruler using an Epson Perfection V300 scanner at 600 dpi (Fig. 1B). Sepal traits (area, length, width), nectary area, petal blade length, and pistil length were measured from the scans in FIJI (Schindelin et al. 2012). The petal nectar spurs and the sepals are the same color in most North American Aquilegia species; therefore, we quantified this color from the sepals only to avoid detecting the contrasting petal blade color, and will hereon refer to it as 'sepal color.' To quantify it, the sepals were cropped from the whole scan and the background was removed in Adobe Photoshop; RGB values were measured using MATLAB (2019b) and averaged across all pixels in each scan; and the average RGB values were converted to the three-dimensional CIE $\mathrm{L}^{*} \mathrm{a}^{*} \mathrm{~b}^{*}$ color space in $\mathrm{R}$ using the convertColor command ( $\mathrm{R}$ core team 2020). The a* axis represents variation in redgreen, $b^{*}$ represents blue-yellow, and L* light-dark. 

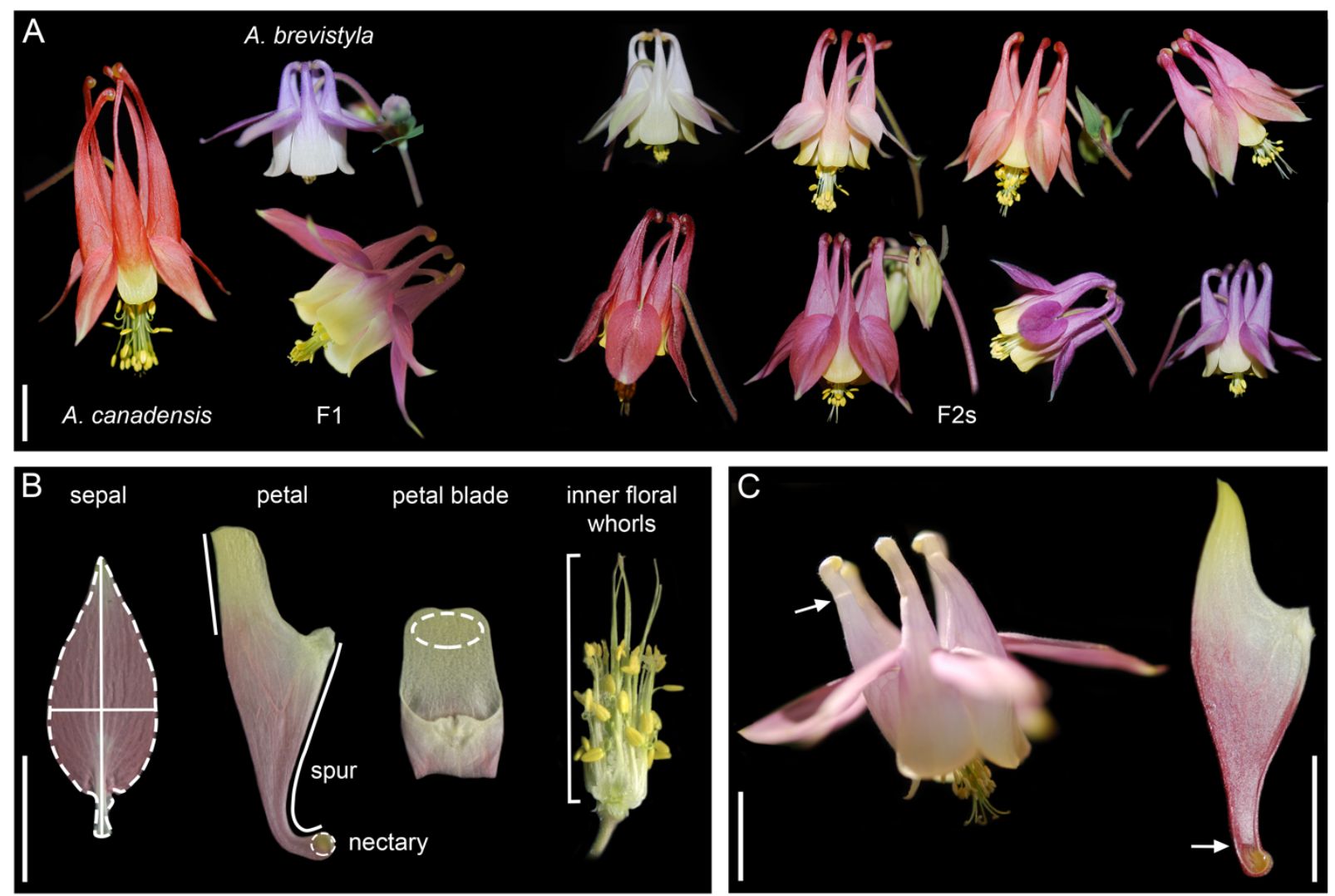

152 Figure 1. Mapping population and phenotyping. A. Parent species A. canadensis and A. brevistyla,

153 an F1 resulting from their cross, and a selection of the 352 members of the F2 population

154 highlighting its phenotypic diversity. B. Morphological and color traits were measured from floral

155 organ scans of three flowers per F2 plant. Example floral organ scans and the phenotypes they

156 produced are shown. Sepal scans were used to determine their area, length, width, and CIE L* a*

$157 \mathrm{~b}^{*}$ color values; petal scans were used to determine blade length, spur length, spur curvature, and

158 nectary area; petal blade scans were used to determine the blade CIE L* a* b* color values; and

159 scans of the inner whorls of floral organs were used to determine pistil length from attachment

160 point to stigma tip (bracket). C. Nectar volume and concentration were measured in all five petals

161 from three flowers per F2 plant, and total sugars were calculated from those values. Petals were

162 dissected longitudinally and nectar (arrows) was collected using a capillary tube. Scale bars $=1 \mathrm{~cm}$. 
164 attachment point and the spur tip were extracted from each petal scan in ImageJ. The petal RGB

165 image was then filtered and binarized using the 'imgaussfilt' and 'imbinarize' functions in

166 MATLAB. A smooth curve representing the petal spur was then obtained using the

167 'bwboundaries' function in MATLAB, with the extracted feature coordinates used for guiding

168 the segmentation. The spur length was then quantified by the total length of the curve segment

169 between the spur tip and the attachment point. The spur curvature quantification was done by

170 computing the curvature difference between the curve segment of each F2 petal and that of a

171 straight reference petal from an A. canadensis flower. For each petal, the signed curvature

$172 k(s)=\frac{x^{\prime}(s) y^{\prime \prime}(s)-x^{\prime \prime}(s) y^{\prime}(s)}{\left(x^{\prime}(s)^{2}+y^{\prime}(s)^{2}\right)^{3 / 2}}$ (Do Carmo 2016) was first computed, where the first zero of the

173 curvature near the spur tip was parameterized as $s=0$ and the attachment point was

174 parameterized as $s=1$. For the computation of $k(s)$, the curve segment was rescaled to be with

175 unit length in order to remove the effect of size on the curvature quantification. The spur

176 curvature was then quantified by the $L^{2}$-norm of the difference between the signed curvature

$177 k(s)$ of the F2 petal and that of the straight reference petal, evaluated from $s=0$ to $s=0.5$ (ie,

178 the lower half of the spur). Note that the part with $s \geq 0.5$ (the upper half of the spur) was

179 excluded as the variation in shape near the attachment point is irrelevant to the spur curvature.

180 The second set of flowers were selected at random from the inflorescence. The petals

181 were taken off the flower and their nectar spurs were removed just below the attachment point.

182 The blades were taped adaxial side up and scanned as for the first batch of flowers. Color was

183 quantified as for the sepals, but instead of sampling across the entire organ, only the distal

184 portion of the blade was used to avoid capturing the spur color, which is identical to the sepal. 
The third set of flowers were also selected at random. Each spur was slit longitudinally at

186 the attachment point for easier access to the nectar, which was collected from each petal

187 individually using a capillary tube (Fig. 1C). Height in the tube was measured to the nearest

$1880.5 \mathrm{~mm}$, the nectar was transferred to a hand-held refractometer (Eclipse Low Volume Nectar

189 Refractometer, 0-50 \& 45-80, Bellingham \& Stanley), and the ${ }^{\circ}$ Brix measurement (sugar

190 concentration by mass) was recorded. All nectar measurements were taken between 9am and

191 noon to minimize circadian variation in nectar traits. Total nectar volume per flower was

192 calculated from the nectar height measurements and the circumference of the capillary tube, and

193 the sugar concentration data were adjusted based on the temperature of the room at the time the

194 measurement was taken according to the manufacturer's instructions. While we do not know the

195 sugar type data for A. brevistyla, the dominant sugar in A. canadensis nectar is sucrose (Macior

196 1978). Total sugars was calculated in $\mathrm{R}$ by fitting a power model to the ${ }^{\circ}$ Brix and molarity data

197 in the Concentrative Properties of Aqueous Solutions Standard Table for Sucrose (CRC

198 Handbook of Chemistry \& Physics 2019); using that model to convert the temperature-adjusted

199 sugar concentration values to molarity; and then multiplying molarity by nectar volume (as in

200 Bolten et al. 1979).

201 For all traits, the final phenotypic value for each F2 is the average phenotypic value of

202 the three flowers after averaging across multiple organs within a flower, where applicable. Not

203 all 352 F2s were phenotyped for all traits: the first set of flowers were prioritized so that the vast

204 majority of F2s have all morphological traits quantified, whereas only 303 F2s produced enough

205 flowers to quantify nectar traits. Parental phenotypes were also collected as for the F2s; the

206 plants that were phenotyped were not the parents themselves, but close relatives (siblings and

207 offspring). Normality of the phenotypic distributions was assessed with the Shapiro-Wilk Test 
208 using the shapiro.test command in R (R core team 2020), and pairwise correlations were tested

209 with the Spearman method using the Hmisc R package (Harrell 2020).

\section{QTL mapping}

All QTL mapping analyses were done using the R package R/qtl v1.46-2 (Broman et al.

213 2003). The genetic map was estimated from F2 genotypic data, which was then combined with

214 the phenotypic data to perform QTL mapping using the Hayley-Knott regression method. The

215 'scanone' function was used to estimate single QTL locations on each chromosome, and

216 'scantwo' was used to determine interactions between QTL pairs. These data were used as inputs

217 for a multiple QTL model, which was assessed and adjusted using the 'fitqtl' and 'refineqtl'

218 commands. The percent variation explained (PVE) for each component of the model and for the

219 model as a whole were estimated using these functions as well. 1.5 LOD intervals of each

220 significant QTL were obtained from the refined model. 1,000 permutations were used to

221 calculate a significant LOD cutoff of 3.5, equivalent to a 5\% false discovery rate. For the nectar

222 traits (volume, sugar concentration, and total sugars), all analyses were performed with a batch

223 covariate to account for the different growing conditions experienced by the two batches of

224 plants.

225

226 Identification of color candidate loci

227 Likely homologs for regulatory and biosynthetic genes involved in producing floral

228 pigments were identified by DELTA-BLAST 2.11.0+ (Boratyn et al. 2012) searches of the

229 predicted Aquilegia proteome (version 3.1, https://phytozome.jgi.doe.gov), with protein

230 sequences of genes characterized in other taxa as input queries (Table S2). Searches were also 
231 run against the predicted Arabidopsis thaliana proteome (TAIR10; Berardini et al. 2015). Blast

232 results were then filtered based on percent identity and alignment length.

234 identified (Fig. S1). Multiple sequence alignment of characterized genes, Aquilegia blast hits,

235 and Arabidopsis blast hits was performed with clustal omega, using default parameters (Sievers

236 et al. 2011). Alignments were evaluated in Jalview (Waterhouse et al. 2009). Poorly aligned

237 sequences lacking conserved elements were removed from the alignment, and, except for

238 alignments of MYBs, positions with a quality score less than 50\% were masked. MYB

239 alignments were manually trimmed to R2R3 or R3 regions (Stracke et al 2001.) and insertions

240 were masked. Neighbor joining trees were then constructed based on BLOSUM62 distances

241 (Jalview; Fig. S1).

\section{Results}

$244 \quad \underline{\text { Phenotypic data }}$

In our F2 population of 352 individuals, we analyzed measurements of 17 traits that

246 captured floral color, nectar composition, and organ morphology in order to understand trends in

247 phenotypic distribution, relationship to parental phenotypes, and correlations between traits. The

248 three sepal size traits were normally distributed, as were spur length and blade CIE $\mathrm{b}^{*}$ and CIE

249 L* (Fig. 2, S2). All other traits were non-normally distributed. In general, the traits exhibited

250 unimodal distributions except for the three sepal color CIE axes and sugar concentration, which

251 were bimodally distributed. We found that roughly half of the F2 trait values fell predominantly

252 between parental means (spur curvature, spur length, blade length, pistil length, sepal and blade

253 CIE $b^{*}$ ) while the other half exhibited transgressive segregation (sepal area, length, and width; 
bioRxiv preprint doi: https://doi.org/10.1101/2021.04.12.439277; this version posted April 13, 2021. The copyright holder for this preprint (which was not certified by peer review) is the author/funder, who has granted bioRxiv a license to display the preprint in perpetuity. It is made available under aCC-BY-NC-ND 4.0 International license.
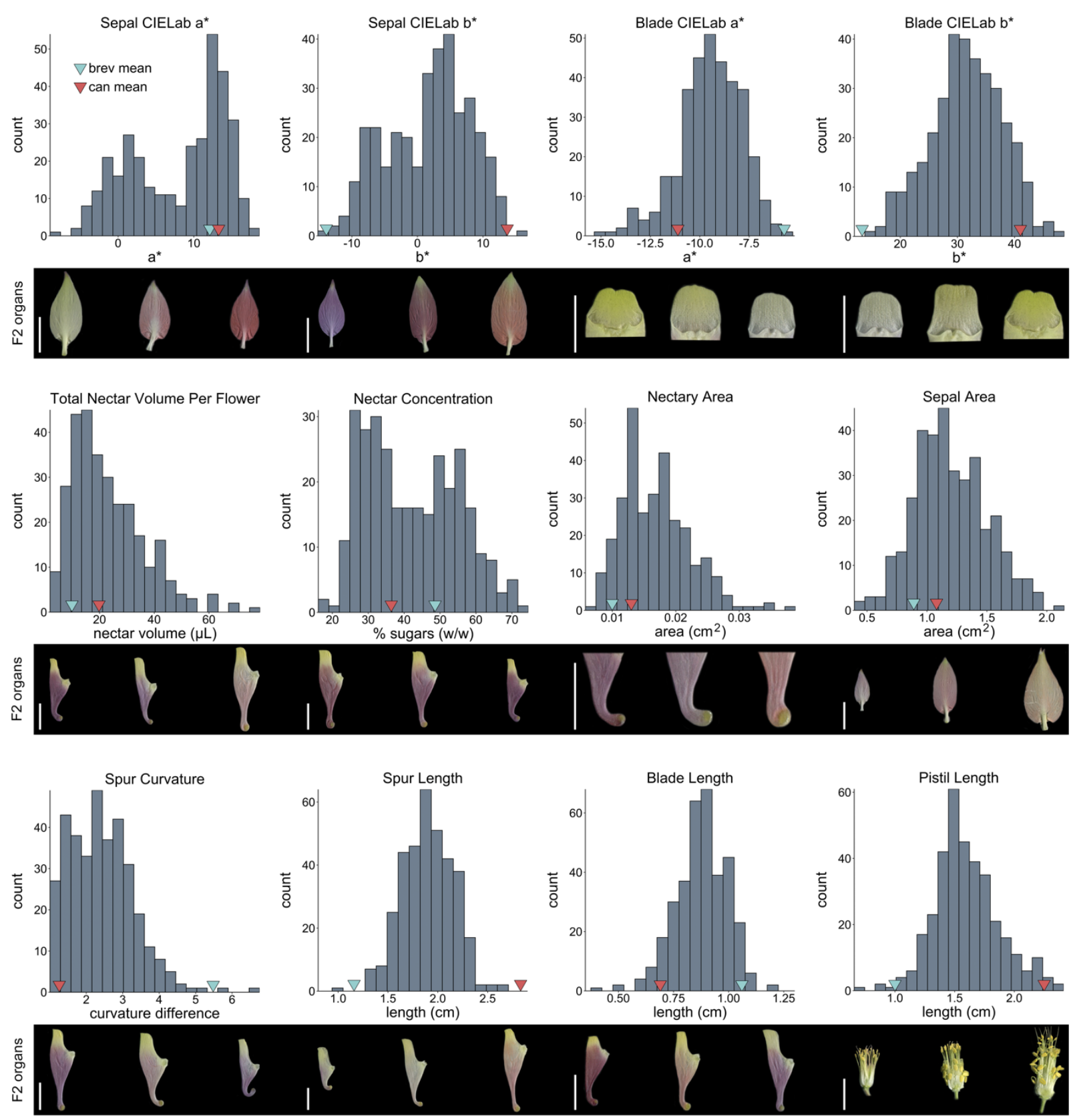

256 Figure 2. F2 population floral trait histograms (remainder in Fig. S2). Blue and red arrows mark

257 the phenotypic means of $A$. brevistyla (brev mean) and A. canadensis (can mean) plants that

258 were closely related to the parents. Representative floral organs from F2s with the lowest, mean,

259 and highest phenotypic values are shown below each histogram. Scale bars $=1 \mathrm{~cm}$. 
sepal and blade CIE a* and CIE L*; nectary area; and all of the nectar traits).

Correlations were common both among and between morphological and biochemical

263 measured within the same organ (Table 1). Sepal color CIE L* and CIE a* topped the list (-0.95,

$264 p<0.001$ ), followed closely by sepal size traits (sepal area and width, $0.92, p<0.001$; sepal area

265 and length, $0.87, p<0.001)$. Nectar traits exhibited the next highest correlation coefficients with

266 nectar volume and concentration at $-0.80(p<0.001)$, and nectar volume and total sugars at 0.79

$267(p<0.001)$. The highest correlation in organs of different types was between pistil length and 268 spur length $(0.71, p<0.001)$. Although all pairwise combinations of CIE $\mathrm{L}^{*} \mathrm{a} * \mathrm{~b} *$ values were

269 significantly correlated for both sepal and blade color, very different correlation patterns

270 emerged in the sepal and blade colors when we plotted these phenotypes. In blades, the

271 phenotypes form a single continuous cluster with yellower blades having low CIE a* and high

272 CIE*b values, and whiter blades having high CIE $\mathrm{a}^{*}$ and low CIE $\mathrm{b}^{*}$ values (Fig. 3, top). In

273 sepals, however, two clusters are present (Fig. 3, bottom): one in the high CIE b*, low CIE a*

274 quadrant that appear mostly white and yellow, and the other in the high CIE a* half that have

275 pigment ranging from red (high $\mathrm{CIE} \mathrm{b}^{*}$ ) to blue (low CIE $\mathrm{b}^{*}$ ). Given these patterns, correlations

276 between single CIE axes and morphological traits could be informative for blade color, but not

277 for sepal color, because a high CIE a* value for blades consistently means that the blades are

278 white, while sepals with high CIE a* values could be white, red, or anything in between.

280 Genotypic data

281 Sequence reads at 200,619 SNP positions across 671 marker bins, averaging 299 SNP positions 282 per bin, were used to genotype the F2 population. Several bins had very few SNP positions $(<33$ 


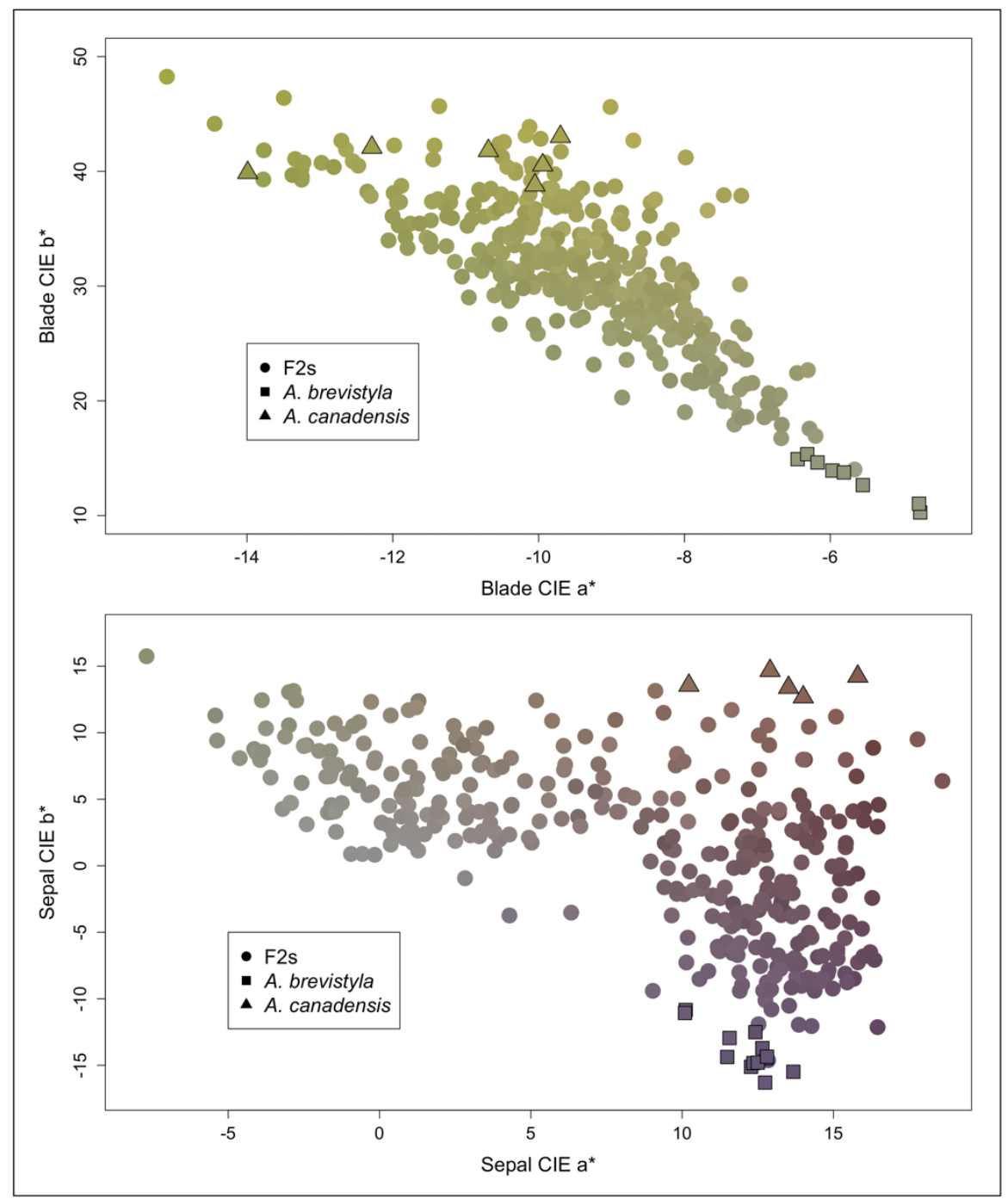

284 Figure 3. Plots of blade color CIE a* and CIE b* (top) and sepal color CIE a* and CIE b* (bottom),

285 exhibiting different correlation patterns. Points are color-coded based on the mean RGB values of

286 the blade or sepal for that individual F2 or parent plant.

288 loci/bin) making genotyping difficult, with the result that only 620 markers were used to estimate

289 the genetic map. Genetic ordering of markers is largely consistent with the physical assembly of

290 the A. coerulea "Goldsmith" reference genome sequence, however, similar to what has been seen

291 in other genetic crosses in Aquilegia, several markers mapped to genetic locations inconsistent 
292 with the physical map, suggesting that there may be some physical differences between the

293 individual used for assembling the reference genome and other species (Fig. S3; Filiault et al.,

294 2018, Ballerini et al., 2020). Overall, patterns of recombination across chromosomes in this cross

295 mimic those seen in other Aquilegia crosses, whereby recombination rate is much higher at

296 chromosome tips but rarely occurs across large physical spans in the middle of chromosomes

297 (Fig. S3). The cross exhibits a substantial amount of transmission ratio distortion, overall

298 favoring $A$. canadensis alleles, possibly because $A$. canadensis pollen has evolved to travel down

299 longer styles than that of $A$. brevistyla. Loci homozygous for $A$. canadensis alleles are greatly

300 overrepresented across most of chromosome 1 (chr1), and moderately overrepresented in regions

301 of chrs 3, 5, and 6 (Fig. S4). A. brevistyla homozygotes are strongly overrepresented on one end

302 of chr2 (Fig. S4).

QTL mapping

We identified a total of 66 QTL for the 17 traits mapped in this study, with 2-6 significant QTL per trait (Table 2, Fig. 4, S5). However, not all 66 QTL are unique; for example, all three

307 CIE axes for sepal color have the same significant QTL at 48.3cM on chr1. We define a major 308 effect QTL as one that explains $25 \%$ or more of the variance in the F2 population (Bradshaw et 309 al. 1995), and a QTL of moderate effect as one with 10-25\% variance explained (PVE). With 310 this framework in mind, a single major locus and four minor loci control the sepal color (CIE $311 \mathrm{~L} * \mathrm{a} * \mathrm{~b} *$ as a whole), while QTL on every chromosome contribute to blade color and vary from 312 minor to moderate, and moderate to major, depending on which axis of the CIE L*a*b* color 313 space is being mapped. The multiple QTL model for sepal color had the highest overall PVE in 314 the study (87 for CIE L*, 81.7 for CIE a*, 87.3 for CIE $b^{*}$ ). The QTL for the nectar traits had the 
315 lowest overall PVEs, with each explaining less than $10 \%$ of the variance in the F2s. We included

316 a batch covariate in the QTL models for nectar traits to account for the different growth

317 conditions in the two greenhouses; this covariate explained $35 \%$ of the variance in nectar volume

318 and $51.2 \%$ of the variance in concentration, but only $5.8 \%$ of the variance in total sugars. The

319 multiple QTL models for morphological traits had overall PVEs ranging from 22.6 for sepal

320 length, to 74.4 for pistil length, and had equally variable genetic architectures: a single major

321 locus and two minor ones contribute to spur curvature, while the maps of blade length and spur

322 length contain six loci of minor to moderate effect. No QTL interactions were detected except in

323 the maps for sepal color, where the major locus on chr1 interacts with the minor loci on chrs 2, 4,

324 and 6.

325 All alleles at identified QTL were in the direction of parental divergence except for single

326 loci in the maps for sepal length (chr5), sepal CIE a* and CIE L* (chr1), blade CIE L* (chr2),

327 spur curvature (chr4), nectar volume (chr4), total sugars (chr2), and nectary area (chr4). All

328 transgressive traits except sepal color exhibited overdominance at one or more loci, most notably

329 the locus on chr2 for total sugars and chr3 for sepal length.

330 QTL co-localization, defined by overlapping 1.5 LOD intervals, was abundant in this

331 dataset (Fig. 4). All of the sepal size loci co-localize, with the exception of the unique chr5 locus

332 for sepal length. The loci on chrs 2, 6, and 7 for sepal size overlap with sepal color loci. Of the

333 six QTL identified for blade length, four overlap with loci for blade color and spur length. The

334 three nectar composition traits and nectary area each have 2-3 peaks on chrs 2, 3, 4, or 7, all of

335 which co-localize. Notably the large peaks on chr7 for spur length and spur curvature are not

336 colocalized, although their peaks are less than 12cM apart. The QTL on chrs 1, 2, 3, and 6 are 
bioRxiv preprint doi: https://doi.org/10.1101/2021.04.12.439277; this version posted April 13, 2021. The copyright holder for this preprint (which was not certified by peer review) is the author/funder, who has granted bioRxiv a license to display the preprint in perpetuity. It is made available under aCC-BY-NC-ND 4.0 International license.

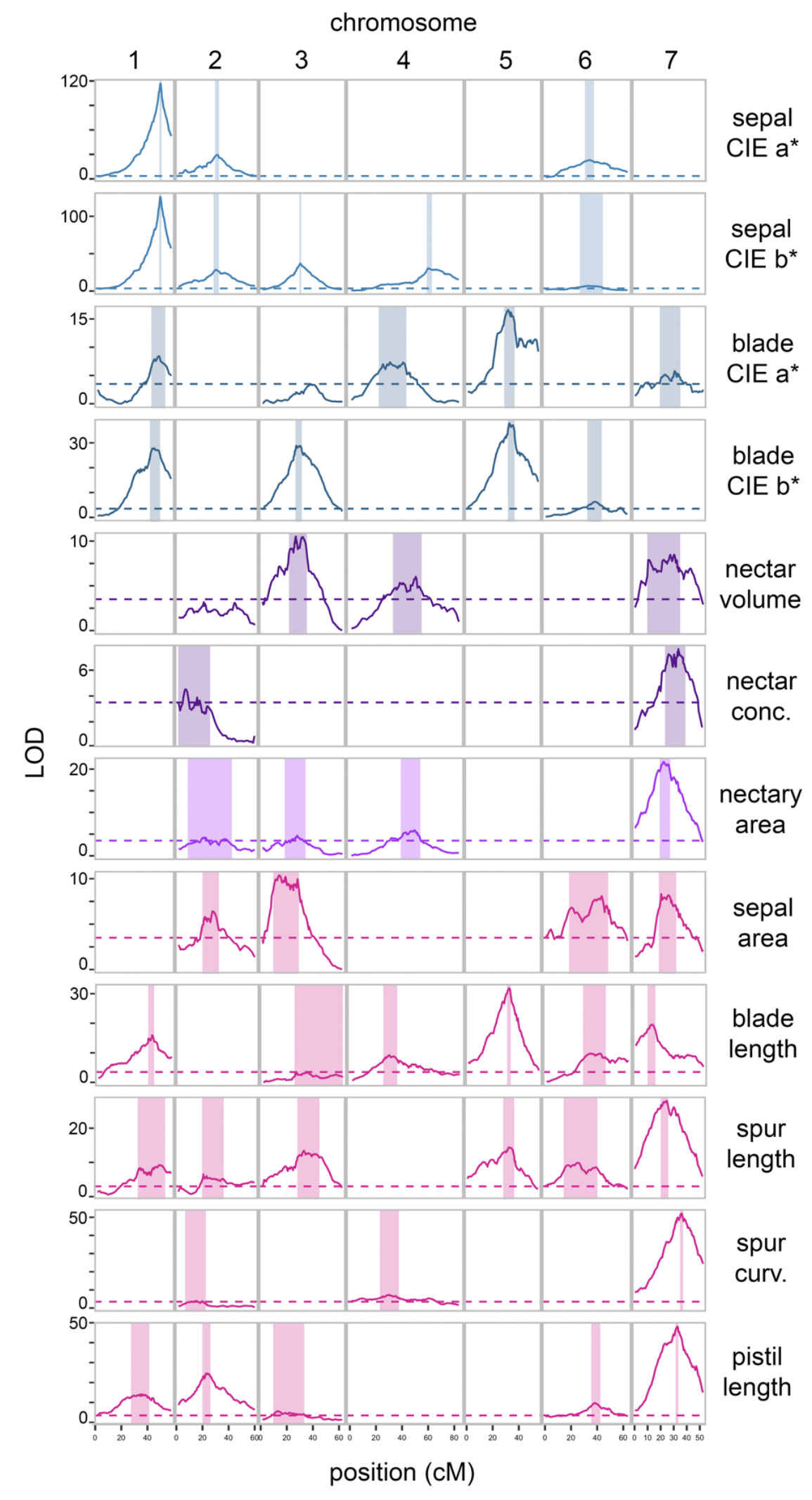

338 Figure 4. Floral trait QTL maps (remainder in Fig. S5). Dashed line represents the significant 
LOD cutoff of 3.5, shaded areas represent the 1.5 LOD interval for each peak. Abbreviations:

341 conc., concentration; curv., curvature.

colocalized between spur length and pistil length, and their large peaks on chr7 are separated by $5 \mathrm{cM}$. The nectar peaks co-localize with those of spur length and pistil length on chrs 2,3 , and 7 . intervals of the morphological trait QTL, we will not speculate about potential causative loci

349 here because characterization of the developmental basis of these traits is still ongoing and not

350 all of these genes have been functionally validated in other systems. The genetic basis of floral

351 color, on the other hand, has been well-characterized in many systems (reviewed in Tanaka et al.

352 2008), and based on this understanding we built an extensive catalog of likely homologs of floral

353 pigment-related genes (Table S3-S4, Fig. S1). The molecular underpinnings of nectar synthesis

354 are becoming clearer, with multiple key genes having been recently characterized (Chen et al.

355 2010; Lin et al. 2014; Min et al. 2019). We identified many of these known genetic players in

356 color biosynthetic pathways and nectar composition in the 1.5 LOD intervals of QTL

357 contributing to these respective traits.

$358 \quad$ Floral pigment analyses done by Taylor (1984) identified carotenoids and anthocyanins

359 in $A$. canadensis, and anthocyanins (but no carotenoids) in lavender-blue species similar to $A$.

360 brevistyla. We identified Aquilegia homologs of known members and regulators of the

361 anthocyanin biosynthetic pathway (ABP) and the carotenoid biosynthetic pathway (CBP)

362 beneath peaks in the sepal color maps (sepal CIE L*, a*, and $b^{*}$ maps collectively). The blade 
363 color maps only contained CBP Aquilegia homologs, consistent with the lack of anthocyanins in

364 the blades of the parental species.

Two core members of the ABP and a family of modification enzymes appear to be controlling anthocyanin production in this cross (Tanaka et al. 2008; Matsuba et al. 2010; Table S3). FLAVONOID 3',5'-HYDROXYLASE (AqF3'5'H), a major branching enzyme in the ABP, is under the large-effect QTL on chr1; several duplicates of GLYCOSYL HYDROLASE (AqGH), a vacuolar anthocyanin modification enzyme, are under the chr2 locus; and DIHYDROFLAVONOL REDUCTASE (AqDFR), another member of the ABP downstream of $F 3^{\prime} 5^{\prime} H$, is under the peak on chr6. The minor peak on chr4 is the only sepal color QTL for which we could not identify a gene known to be involved in the ABP. are found in the 1.5 LOD intervals of the color QTL and could be candidates for contributing to carotenoid dynamics in this cross. CBP regulators CAROTENOID CLEAVAGE DIOXYGENASE 4.1 and 4.2 (AqCCD4.1 and $A q C C D 4.2)$ are under the chr3 locus shared between the sepal and blade color maps (Ohmiya 2009), as is ORANGE PROTEIN (AqOR), a gene that affects carotenoid accumulation ( $\mathrm{Li}$ et al. 2001). Except for the minor locus on chr4, all of the QTL specific to blade color each contain 1-5 genes known to be involved in carotenoid synthesis or regulation (summarized in Table S4). contains AqLRP, a member of the STYLISH (STY) gene family that is necessary for nectary

383 development in the Ranunculaceae (Min et al. 2019). The locus on chr3 in the nectar volume, 384 total sugars, and nectary area maps contains an Aquilegia homolog of a member of the SWEET 385 family, bidirectional sugar transporters involved in nectar secretion (Chen et al. 2010). The chr7 
and chr4 loci each contain several sugar transporters and sucrose synthesis genes; unique to the sugar concentration chr7 1.5 LOD interval is SUCROSE PHOSPHATE SYNTHASE-1 (SPSIF), a sucrose synthesis gene that is necessary for nectar production in Arabidopsis (Lin et al. 2014).

\section{Discussion}

We identified QTL for multiple complex traits related to pollination syndromes in $A$. canadensis and A. brevistyla. These traits cut across well-defined genetic pathways such as anthocyanin biosynthesis, as well as those that are less understood such as organ size and shape.

\section{$\underline{\text { Color }}$}

Anthocyanin pathway

A. canadensis flowers make red cyanidin and pelargonidin anthocyanidin pigments, while lavender-blue species like $A$. brevistyla only make delphinidins (Taylor 1984). The QTL maps for sepal color consist of a single locus of major effect that interacts with several loci of minor

400 effect: we have identified $A q F 3{ }^{\prime}{ }^{\prime} H$ at the large QTL on chr1, and $A q G H$ and $A q D F R$ at the

401 minor loci on chrs 2 and 6, respectively, as good candidate loci for controlling color variation.

402 Here we present a model for how the interaction of these candidate loci may contribute to sepal 403 pigmentation in this cross, which categorizes the F2s into three classes based on their phenotypes 404 and genotypes at these loci (summarized in Fig. 5). $A q F 35^{\prime} \mathrm{H}$ is likely the primary cause of the shift from blue to red in this cross, and has

406 also been implicated in blue-to-red color shifts in Phlox, Penstemon, and Iochroma (Hopkins and 407 Rausher 2011; Smith and Rausher 2011; Wessinger and Rausher 2013). Expression of $F 3$ '5'H 408 diverts anthocyanin precursors down the branch of the ABP that produces blue delphinidins, 
409 while lack of $F 3^{\prime} 5^{\prime} H$ expression yields red pelargonidins and cyanidins (reviewed in Tanaka et

410 al. 2008). Class $1 \mathrm{~F} 2 \mathrm{~s}$ are defined by having at least one $A$. brevistyla $A q F 3$ ' 5 H allele, and

411 clustering in the high CIE a*, low CIE $\mathrm{b}^{*}$ range of the sepal color space (Fig. 5, Class 1 and

412 lower panel). Class 1 F2s are all blue-purple in color, and we believe they are primarily

413 producing delphinidin pigments due to $F 3^{\prime} 5^{\prime} H$ 's ability to outcompete $F 3^{\prime} H$ for substrate (Gerats

414 et al. 1982; Fig. 5, Class 1). Because A. canadensis does not make delphinidins, we assume that

415 its $F 3^{\prime} 5^{\prime} \mathrm{H}$ allele is either non-functional or not expressed, and that in plants homozygous for $A$.

416 canadensis at $A q F 33^{\prime}{ }^{\prime} \mathrm{H}$, flux is directed down the other branches of the ABP.

However, there is substantial phenotypic variation among F2s homozygous for $A$.

canadensis at $A q F 3$ '5 $\mathrm{H}$ : some are pigmented pink-red and have varying CIE a* values but

419 cluster in the high CIE b* range, while others appear to lack anthocyanins and are pure white or

420 yellow, with low CIE a* and high CIE* b values (Fig. 5, bottom panel). We divide these F2s into

421 Class 2 (pigmented pink-red) and Class 3 (white or yellow), and propose that $A q F 3$ '5H

422 epistatically interacts with $A q D F R$ and $A q G H$ to produce these transgressive Class 3 phenotypes.

423 All Class 2 F2s have at least one $A$. canadensis allele at both $A q D F R$ and $A q G H$, while Class 3

424 F2s are homozygous A. canadensis at either or both loci (Fig. 5, Class 2 and Class 3).

426 dihydroflavonols into leucoanthocyanidins, and can have strict substrate specificity for a

427 particular dihydroflavanol (i.e., dihydrokaempferol (DHK), dihydroquercitin (DHQ), or

428 dihydromyricetin (DHM); reviewed in Tanaka et al. 2008). All F2s that are homozygous for $A$.

429 canadensis alleles at $A q F 3$ '5 $\mathrm{H}$ and homozygous for $A$. brevistyla alleles at $A q D F R$ appear to

430 lack anthocyanins (Fig. 5, Class 3; Fig. S6), suggesting that the A. brevistyla protein is unable to

431 process DHQ or DHK, or is outcompeted by other flavonoid pathway enzymes, as has been 


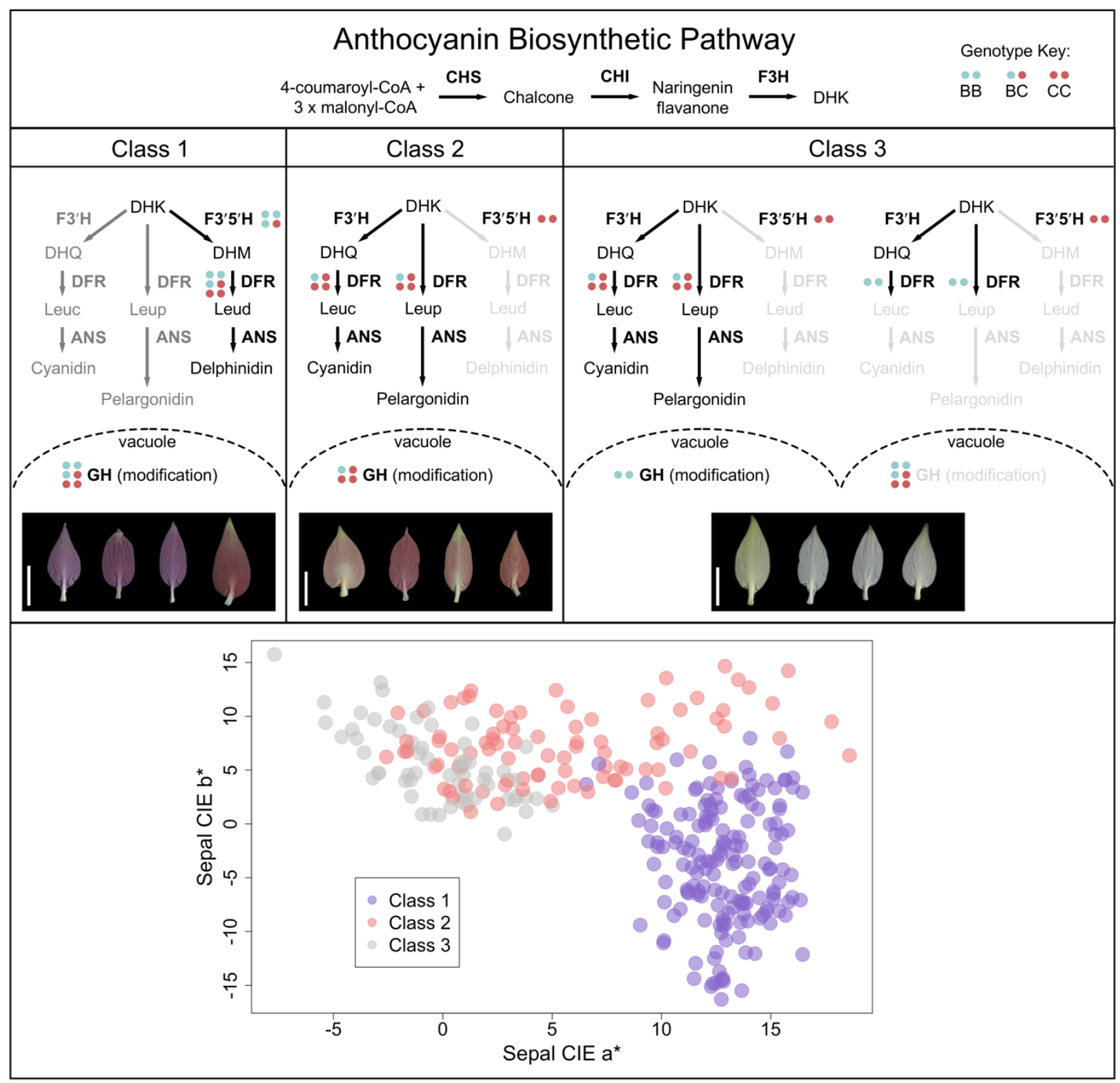

433 Figure 5. Model of anthocyanin dynamics in this cross depicting three classes of pigmentation in

434 the F2s. The header panel shows the first steps of the core ABP, while each Class panel (1-3)

435 shows the later branching steps of the ABP and downstream GH modification, which occurs in

436 the vacuole. Enzymes are in bold and ABP branches that experienced reduced or no flux are in

437 dark grey and light grey, respectively. Allelic genotypes of key enzymes are represented by pairs

438 of blue (A. brevistyla allele) and red (A. canadensis allele) dots. The left Class panel shows Class 
4391 of F2 anthocyanin pigmentation; F2s with an $A$. brevistyla $F 3^{\prime} 5^{\prime} \mathrm{H}$ allele are all pigmented

440 blue-purple due to flux primarily moving through the delphinidin branch of the pathway, and all

$441 A q D F R$ and $A q G H$ genotypes accepting substrates from that branch. The middle Class panel

442 shows Class 2: F2s homozygous for the $A$. canadensis $F 3^{\prime} 5^{\prime} H$ allele are pigmented pink-red

443 because flux is moving through the pelargonidin and cyanidin branches, and they have $A$.

444 canadensis $A q D F R$ and $A q G H$ alleles that are compatible with those substrates. The right Class

445 panel shows Class 3: white and yellow F2s lack anthocyanins because they are homozygous $A$.

446 canadensis at $A q F 3{ }^{\prime}{ }^{\prime} \mathrm{H}$ and homozygous $A$. brevistyla at either $A q D F R$ or $A q G H$, which can

447 only process substrates from the delphinidin branch. Representative sepals from each class of

448 pigmentation and corresponding genotypes are shown below the ABP summaries in each Class

449 panel. Scale bar $=1 \mathrm{~cm}$. The bottom panel shows how these three classes cluster in the CIE a*

450 and $\mathrm{b}^{*}$ color space. $\mathrm{CHS}$, chalcone synthase; $\mathrm{CHI}$, chalcone isomerase; $\mathrm{F} 3 \mathrm{H}$, flavanone 3-

451 hydroxylase; DHK, dihydrokaempferol; F3'H, flavonoid 3'-hydroxylase; F3'5'H, flavonoid 3',5'-

452 hydroxylase; DHQ, dihydroquercitin; DHM, dihydromyricetin; Leuc, leucocyanidin; Leup,

453 leucopelargonidin; Leud, leucidelphinidin; DFR, dihydroflavonol reductase; ANS, anthocyanidin 454 synthase; $\mathrm{GH}$, glycosyl hydrolase.

456 shown in Petunia and Mimulus (Davies et al. 2003; Yuan et al. 2016). In addition to color

457 variation produced by generating different anthocyanidin pigment types, further diversity of

458 floral color is in large part due to modification of anthocyanidin pigments with glycosyl, acyl,

459 and methyl groups. The $A q G H s$ on chr2 are homologues of one such modification enzyme,

460 known to act in the vacuole to glucosylate anthocyanidins in Carnation as well as Delphinium,

461 which is in the same family as Aquilegia (Matsuba et al. 2010; Table S3). In this cross, $A$. 
canadensis $A q G H$ alleles result in redder sepals than $A$. brevistyla alleles, regardless of genotype

463 at $A q F 3{ }^{\prime}{ }^{\prime} \mathrm{H}$ and resulting anthocyanin type (Fig. S7). However, flowers that are homozygous $A$.

464 canadensis at $A q F 3$ '5 $H$ and have at least one $A$. canadensis allele at AqDFR, but are

465 homozygous $A$. brevistyla at $A q G H$, are white-yellow. (Fig. 5, Class 3; Fig. S8). In this case,

466 anthocyanidins were presumably formed and transported into the vacuole, but the A. brevistyla

$467 A q G H$ proteins somehow fail to prevent pigment degradation. The GH family is involved in

468 pigment stability (Sasaki and Nakayama 2015), but we do not have enough information to

469 speculate about an exact mechanism in this cross. Broadly, we can say that in our model, $A$.

470 brevistyla $A q D F R$ and $A q G H$ alleles are only able to act on dihydroflavonols and anthocyanidins

471 produced by the $A q F 3{ }^{\prime} 5 \mathrm{H}$ branch, whereas $A$. canadensis alleles have less specificity and are

472 able to act on all precursors and pigments regardless of which ABP branch produced them.

474 in a dramatic change in phenotype, as in Penstemon, where $F 35^{\prime} \mathrm{H}$ alone is responsible for the

475 blue-to-red transition in the genus (Wessinger et al. 2014). The genetic architecture of blue-to-

476 red shifts can be more complicated, however, as in Iris, where 8 loci contribute to floral color

477 (Brothers et al. 2013). The color shift in the present study is similar to that of Iochroma, which

478 also involves $F 3^{\prime} 5^{\prime} H$ and a change in substrate specificity at DFR (Smith and Rausher 2011), but

479 in Iochroma it was not certain if the change in DFR was absolutely necessary for the transition

480 from blue to red. In this cross, $A q D F R$ and the $A q G H s$ did need to lose their substrate specificity

481 during the transition to hummingbird pollination in order for A. canadensis to evolve its red

482 pigmentation. 


\section{Carotenoid pathway}

A. canadensis flowers contain yellow-orange carotenoid pigments, and species with white petal blades like those of $A$. brevistyla do not (Taylor 1984). While carotenoids are very apparent in the bright yellow petal blades of $A$. canadensis, they also contribute to the red coloration throughout the rest of the flower; indeed, CBP genes appear in the QTL maps for sepal color.

490 The combination of anthocyanins and carotenoids underlying red floral color has been found in 491 other systems including Mimulus and Solanaceae (Bradshaw and Schemske 2003; Ng and Smith 492 2016).

Carotenoid genetic architecture in this system is more complicated than that of the

494 anthocyanins, consisting of multiple loci of small to moderate effect, many of which themselves

495 contain multiple genes known to be involved in the CBP (Table S4). The pleiotropic nature of

496 the CBP and the gaps in our knowledge about carotenoid regulation make it difficult to

497 synthesize these loci into a detailed model such as that presented for the ABP above (Tanaka et

498 al. 2008; Zhu et al. 2010). The sheer number of CBP candidate genes we identified in the 1.5

499 LOD intervals of blade color QTL precludes us from mentioning them all, so we will focus our

500 discussion on the largest peaks on chrs 5,1 , and 3.

The largest-effect peak is on chr5 and contains $A q Z E P$, which acts late in the CBP to

502 convert zeaxanthin into other yellow and orange pigments (Zhu et al. 2010). Silencing of ZEP in

503 California poppy results in zeaxanthin accumulation in the petals far exceeding that in wild type

504 plants and an accompanying shift in color from bright orange to yellow (Zhou et al. 2018).

505 However, AqZEP is at the edge of the 1.5 LOD interval for the chr5 QTL; closer to the peak

506 marker bin are CBP precursors HYDROXYMETHYLBUTENYL DIPHOSPHATE REDUCTASE

$507(A q H D R)$ and several copies of GERANYLGERANYL DIPHOSPHATE SYNTHASE (AqGGPPS; 
508 Table S4). In addition to carotenoid pigments, these genes generate precursors for metabolites

509 essential for plant functioning such as chlorophyll and various hormones (Beck et al. 2013;

510 Botella-Pavía et al. 2004). Due to the high pleiotropic potential of single-copy $A q H D R$ we think

511 it an unlikely candidate for CBP regulation in this cross, but given the multiple copies of

$512 A q G G P P S$, it is possible that one is functioning specifically in floral tissue (Ament et al. 2006).

513 The CIE a* and CIE $\mathrm{b}^{*}$ blade color maps have a peak on chr1 that contains a WRKY that

514 has been found to positively regulate CCD4 in Osmanthus (Han et al. 2016); a potential shared

515 carotenoid regulatory mechanism between such disparate angiosperm genera is fascinating and

516 will require more investigation. $A q C C D 4.1$ and $A q C C D 4.2$, as it happens, are underneath the

517 peak on chr3 that is shared between the blade and sepal color maps. CCD4 regulates carotenoid

518 accumulation by cleaving the pigments into apocarotenoids (Ohmiya 2009), and has been

519 implicated in white/yellow petal color changes in multiple systems, primarily from horticultural

520 cultivars and food crops such as Petunia (Kishimoto et al. 2018), azalea (Ureshino et al. 2016),

521 and Chinese kale (Zhang et al. 2019). Also in the chr3 1.5 LOD interval and closer to the peak

522 marker bin than $A q C C D 4.1 / 2$ is the Aquilegia homolog of $O R$, which was first characterized in

523 cauliflower: a mutation in the gene causes the curd and other vegetative tissues to accumulate

524 large amounts of $\beta$-carotene (Li et al. 2001). The exact mechanism has not been determined but

$525 O R$ is thought to be involved in the formation of a lipoprotein structural sink for the pigments.

$527 \quad$ Nectar

528 Studies into the genetic basis of nectar and nectaries are becoming more common, but the

529 fact that floral nectaries have evolved multiple times across the angiosperms makes it difficult to

530 assess the extent to which their genetic underpinnings are conserved (reviewed in Roy et al. 
531 2017). Another complicating factor is that the quantity and composition of floral nectar is

532 determined by the interaction of development, physiology, and the environment. If we start by

533 considering the parents, $A$. brevistyla and $A$. canadensis, the pollination syndrome literature

534 would lead us to expect that the bee-pollinated species would produce lower-volume, higher-

535 concentration nectar compared to the hummingbird-pollinated sister species (Baker 1975; Gegear

536 et al. 2017). While our parental phenotype data seem to align with this trend, we hesitate to say

537 that it is a definitive example due to the fact that the parents experienced different growing

538 conditions, and nectar is sensitive to environmental variation (Canto et al. 2007). All of the $A$.

539 canadensis plants were grown in the cooler-temperature batch, while the $A$. brevistyla plants

540 were split between the two batches and experienced both high and low temperatures. This is the

541 first study to characterize $A$. brevistyla nectar, but the data from the existing $A$. canadensis nectar

542 literature indicate that nectar volume and sugar concentration can vary widely within and

543 between populations (Mavraganis 1998; Noutsos et al. 2015). Sampling, quantification, and

544 analysis methods are also inconsistent between these studies, indicating a need for

545 standardization of nectar phenotyping practices if we want to be able to make meaningful

546 comparisons across species and studies in the future.

547 In the F2s, the transgressive nature of all four nectar traits suggests that their reshuffled

548 parental alleles released constraints on nectar production present in A. canadensis and A.

549 brevistyla. While there is not a clear pattern of allelic combinations across QTL that correspond

550 to the highest and lowest trait values, the F2s towards the phenotypic maxima and minima do

551 have novel combinations of parental alleles. The environmental variation experienced by the F2s

552 in different growing conditions had a large effect on the nectar traits: the batch covariate of the

553 QTL model explained 35\% of the variance in nectar volume, 51.2\% of the variance in sugar 
554 concentration, and $5.8 \%$ of the variance in total sugars. Despite this, we still found significant

555 QTL for each trait, suggesting that they are genetically robust even under extreme environmental

556 variation. The overall PVE of the QTL model for nectary area (43.1) falls in the middle of the

557 other morphological traits in the study, and has one peak of moderate effect. All nectar traits are

558 significantly correlated with each other in the F2 population, so it comes as no surprise that all

559 nectar trait QTL are co-localized with at least one other's QTL. However, not all QTL are shared

560 among the four traits, so we must examine each trait's relationship to the others in the context of

561 their shared and unique loci.

562 Nectar volume and nectary area have a correlation coefficient of $0.62(p<0.001)$, and

563 their QTL on chrs 3, 4, and 7 have overlapping 1.5 LOD intervals and peaks within a few cM of

564 each other. Nectary area has a QTL on chr2 that appears unique, but the single QTL analysis of

565 nectar volume detected a QTL on that chromosome as well, which only just missed the

566 significant LOD cutoff in the multiple QTL model (Fig. 4, nectar volume). Therefore, we believe

567 that nectar volume in this system is in large part controlled by the amount of secretory tissue

568 present in a nectar spur. Nectary area and nectar volume are strongly correlated in an F2

569 population of a cross between bee-pollinated Penstemon amphorellae and hummingbird-

570 pollinated $P$. kunthii, and nectary area is predictive of pollination syndrome throughout the genus

571 (Katzer et al. 2019). QTL mapping of nectar traits in Ipomopsis also found significant correlation

572 between nectar volume and corolla tube size as well as co-localization of their QTL (Nakazato et

573 al. 2013). These authors hypothesized that this could be due to the effect of nectary area,

574 although they did not measure nectary area directly. It would be fascinating to know if this

575 pattern holds true in other systems and represents a common mechanism for increasing nectar

576 volume during pollinator transitions. The morphology of the Aquilegia nectar spur lends itself to 
relatively easy quantification of nectary tissue area, but phenotyping in other species can be quite complex - Penstemon required dissection and microscopy, for example (Katzer et al. 2019).

Nonetheless, we encourage this extra effort in future studies, for it could greatly increase our understanding of nectar biosynthesis and evolution in the angiosperms.

Nectar volume and sugar concentration have a correlation coefficient of $-0.80(p<0.001)$,

582 but their relationship is more complicated than that of nectar volume and nectary area. On chr7,

583 there is a peak for nectar volume and sugar concentration, but not total sugars. Homozygous $A$.

584 canadensis F2s at that locus have a higher nectar volume than their homozygous $A$. brevistyla

585 counterparts, but also a lower sugar concentration, indicating that the increase in volume is

586 having a diluting effect on the nectar solutes. A very different scenario is playing out on chr3,

587 however, where there is a peak for nectar volume and total sugars, but not for sugar

588 concentration. Homozygous $A$. canadensis F2s at that peak have both a higher nectar volume and

589 total sugars than homozygous $A$. brevistyla F2s, suggesting that whatever mechanism is

590 controlling volume at this locus is not having a diluting effect on the sugar.

There are multiple sugar transport and synthesis genes under each of these peaks, but we

592 do not have the power to distinguish between a single causative locus or multiple tightly-linked

593 ones, and substantial investigation would be required to determine if these candidates are

594 involved in Aquilegia nectar production. Overall, the genetic architecture of nectar traits in

595 Aquilegia is similar to that of Ipomopsis, in which several loci of small effect contribute to nectar

596 volume (Nakazato et al. 2013). This is in stark contrast to Penstemon, Petunia, and Mimulus,

597 where QTL mapping of nectar traits identified single large-effect loci critical to pollinator shifts

598 (Bradshaw et al. 1995; Stuurman et al. 2004; Wessinger et al. 2014). We do know from the

599 Penstemon study that the loci controlling nectar volume and concentration can be totally separate 
600 (Wessinger et al. 2014), while in Mimulus they overlap entirely (Bradshaw et al. 1995);

601 Aquilegia appears to be somewhere in the middle.

602

$603 \quad$ Morphological traits

Two phylogenies support the sister relationship of $A$. brevistyla and $A$. canadensis, with

605 divergence estimated to have occurred less than three million years ago (Whittall and Hodges

606 2007; Fior et al. 2013). Major morphological shifts were required during this transition from bee

607 to hummingbird pollination in order to facilitate successful pollen transfer and pollinator reward.

608 At first glance, A. canadensis flowers are much larger than those of $A$. brevistyla, but closer

609 examination of each floral whorl reveals a more complicated picture. The sepals, which are

610 petaloid in Aquilegia and contribute to pollinator attraction, are actually of a comparable size

611 between the two parents (Fig. 2). The extreme transgressive segregation of sepal size traits in the

612 F2s suggests that the mechanisms maintaining the parents' modest sepal size are not the same

613 and are released in the F2 generation when recombined. The dramatic morphological differences

614 between the parental flowers are concentrated in the inner whorls. $A$. canadensis petal spurs are

615 on average more than twice as long as A. brevistyla spurs and are of a very different shape, but

616 their blades are only half as long. We know from previous work that these two regions of the

617 petal are highly differentiated in terms of gene expression patterns (Yant et al. 2015), and in this

618 study we found variable genetic architectures among the three petal traits. Even though the

619 parental spur sizes are very different, their nectary area is similar; nectary area does however

620 segregate transgressively in this cross, in contrast to the other three petal traits that segregate

621 within their parental extremes. Pistils are also more than twice as long in A. canadensis than $A$.

622 brevistyla, likely an adaptation to promote pollen transfer from the head of a hovering 
623 hummingbird. A fundamental question in this system (and in the radiation of Aquilegia writ

624 large) is how these extreme morphological changes evolved so rapidly.

625 The abundant co-localization of morphological trait QTL (along with color and nectar

626 traits) could hold part of the answer. It certainly explains large correlation coefficients between

627 many phenotype pairs in the F2 population. Genetic correlation, either due to pleiotropy or tight

628 genetic linkage, can facilitate adaptation and speciation because selection on one trait can easily

629 lead to a correlated response in the other trait (Via and Hawthorne 2005; Hermann and

630 Kuhlemeier 2011). Four of the five peaks in the pistil length map co-localize with those in the

631 spur length map, with the exception of the large QTL on chr7, which are only 8cM apart. Four of

632 the blade length loci co-localize with these traits as well, and all three traits are in the direction of

633 divergence: F2s that are homozygous A. canadensis at these loci have longer spurs, longer

634 pistils, and shorter blades than their homozygous $A$. brevistyla counterparts. Especially

635 interesting is the fact that the largest QTL for three morphological traits critical to pollination

636 success are all within a few centimorgans of each other and have matching divergence directions:

637 spur length is at $24.6 \mathrm{cM}$, pistil length at $32.6 \mathrm{cM}$, and spur curvature at $36 \mathrm{cM}$ on chr7.

Nearly all of the morphological traits mapped in this study are controlled by multiple loci

639 of small- and medium-effect, in keeping with the pattern established by previous work in other

640 floral systems in which complex developmental traits have equally complex genetic architectures

641 (Goodwillie et al. 2006; Nakazato et al. 2013; Wessinger et al. 2014). One trait, spur curvature,

642 broke from this norm, with a single locus of major effect on chr7 (as well as two very minor ones

643 on chr 2 \& 4). This supports earlier work by Prazmo (1965), which showed that F2 hybrids of $A$.

644 canadensis and $A$. flabellata exhibit a 3:1 ratio of curved:straight spurs, indicating the presence

645 of just one causative locus. Spur curvature is not the only example of a single locus of major 
646 effect underlying a morphological trait in Aquilegia: the presence of the petal nectar spur itself

647 maps to a single locus $P O P O V I C H$, which encodes a $\mathrm{C} 2 \mathrm{H} 2$-zinc finger transcription factor

648 (Ballerini et al. 2020). Single large loci controlling floral morphological variation remain rare in

649 the QTL mapping literature, such as L02, an HLH transcription factor responsible for style length

650 in Solanum (Chen et al. 2007), as well as a stigma exertion locus in Oryza (Miyata et al. 2007).

651 However, examples of large-effect loci acting in concert with several smaller loci to shape floral

652 organs are quite common. This pattern is found in the QTL maps of sepal length in Iris (Bouck et

653 al. 2007); sepal length, petal width, and pistil length in Arabidopsis (Juenger et al. 2005); and

654 multiple floral organ size traits in Petunia (Hermann et al. 2015).

In this study we characterized the genetic architecture of 17 floral traits encompassing

656 color, nectar composition, and organ morphology that shifted during a bee-to-hummingbird

657 pollinator transition in sister species of Aquilegia. This work represents the first comprehensive

658 QTL analysis of Aquilegia pollination syndromes using genomic data, and will lead to exciting

659 future work such as validating the ABP model, exploring the numerous CBP candidate genes,

660 and characterizing the developmental basis of morphological traits in an effort to clarify potential

661 causative loci. Given that there is a second independent transition to hummingbird pollination in

662 the genus, and the multiple independent gains and losses of carotenoids, opportunities for

663 comparative studies abound. When we synthesize our findings in Aquilegia with previous QTL

664 studies in other systems, we see that every kind of genetic architecture is possible during rapid

665 radiations. This study alone shows that color can be influenced by many loci of small- and

666 moderate-effect, while nectar spur shape can be sculpted by a single locus of large-effect:

667 perhaps it is time to retire the notion of a dichotomy between genetic architectures of

668 biochemical traits and morphological traits. 


\section{Author contributions}

670 ESB conceived of the study, and designed it with MBE. ESB grew, crossed, and sequenced the

671 parental species, and grew and pollinated the F1 individuals. MBE and YM grew the F2s. GPTC

672 and LM developed the spur curvature phenotyping method, which also yielded spur length

673 phenotypes. AD phenotyped nectary area. All other phenotyping was conducted by MBE. ESB,

674 MBE, and YM prepared the F2 sequencing libraries and constructed the genetic map. MBE and

675 ESB performed QTL mapping analyses, with assistance from AD for nectary area. ND identified

676 anthocyanin and carotenoid loci, and developed the anthocyanin model with ESB and MBE.

677 EMK and SAH provided oversight of the study. MBE wrote the manuscript with input from the

678 co-authors.

\section{Acknowledgements}

681 We would like to thank the Arnold Arboretum of Harvard University for their generous

682 accommodation of our plants in their Weld Hill growth facilities and use of lab space while

683 phenotyping; Kea Woodruff and Laura Craig-Comin for their plant pest management expertise;

684 Olivia Meyerson and Michael Shahandeh for their discussions of QTL analysis; Joanna

685 Ladopoulu for her assistance with phenotyping; Natasha Parikh for her guidance with Matlab;

686 Elizabeth McCarthy for her advice about floral color; Karl Broman for answering questions

687 about the R/qtl package and maintaining his Google group on the subject; and the WAWG for

688 their thoughtful manuscript suggestions. This material is based upon work supported by the

689 National Science Foundation Graduate Research Fellowship under grant no. DGE1745303 (to

690 MBE). We acknowledge the Harvard Quantitative Biology Initiative and the NSF-Simons Center

691 for Mathematical and Statistical Analysis of Biology at Harvard, award no. 1764269 (to GPTC 
692 and LM). ESB was supported by the NIH under the Ruth L. Kirschstein National Research

693 Service Award (F32GM103154). Research was funded by the UC Santa Barbara Harvey Karp

694 Discovery award to ESB. The sequencing was carried out by the DNA Technologies and

695 Expression Analysis Cores at the UC Davis Genome Center, supported by NIH Shared

696 Instrumentation Grant 1S10OD010786-01 and the Biological Nanostructures Lab at UC Santa

697 Barbara. We thank Jennifer Smith and the use of the research facilities within the California

698 NanoSystems Institute, supported by the University of California, Santa Barbara and the

699 University of California, Office of the President.

700

701 Data Accessibility Statement

702 All sequence data are deposited in the Sequence Read Archive under BioProject ID

703 PRJNA720109.

704

705 Conflict of Interest Statement

706 The authors have no competing interests relative to this work.

707

708

709

710

711

712

713

714 


\section{Works Cited}

716

Ament, K., C. C. Van Schie, H. J. Bouwmeester, M. A. Haring, and R. C. Schuurink. 2006. Induction of a leaf specific geranylgeranyl pyrophosphate synthase and emission of $(E, E)$ 4,8,12-trimethyltrideca-1,3,7,11-tetraene in tomato are dependent on both jasmonic acid and salicylic acid signaling pathways. Planta 224:1197-1208.

Armbruster, W. S., and N. Muchhala. 2009. Associations between floral specialization and species diversity: Cause, effect, or correlation? Evol. Ecol. 23:159-179.

Baker, H. G. 1975. Sugar Concentrations in Nectars from Hummingbird Flowers. Biotropica $7: 37-41$

Ballerini, E. S., Y. Min, M. B. Edwards, E. M. Kramer, and S. A. Hodges. 2020. POPOVICH, encoding a $\mathrm{C} 2 \mathrm{H} 2$ zinc-finger transcription factor, plays a central role in the development of a key innovation, floral nectar spurs, in Aquilegia. Proc. Natl. Acad. Sci. U. S. A. $117: 22552-22560$.

Beck, G., D. Coman, E. Herren, M. Á. Ruiz-Sola, M. Rodríguez-Concepción, W. Gruissem, and E. Vranová. 2013. Characterization of the GGPP synthase gene family in Arabidopsis thaliana. Plant Mol. Biol. 82:393-416.

Berardini, T. Z., L. Reiser, D. Li, Y. Mezheritsky, R. Muller, E. Strait, and E. Huala. 2015. The arabidopsis information resource: Making and mining the "gold standard" annotated reference plant genome. Genesis 53:474-485.

Bolten, A. B., P. Feinsinger, H. G. Baker, and I. Baker. 1979. On the calculation of sugar concentration in flower nectar. Oecologia 304:301-304.

Boratyn G.M., Schäffer A.A., Agarwala R., Altschul S.F., Lipman D.J., and T. L. Madden. (2012). Domain enhanced lookup time accelerated BLAST. Biology Direct 7:12. 
738 Botella-Pavía, P., Ó. Besumbes, M. A. Phillips, L. Carretero-Paulet, A. Boronat, and M. a key role of hydroxymethylbutenyl diphosphate reductase in controlling the supply of

Bouck, A., S. R. Wessler, and M. L. Arnold. 2007. QTL analysis of floral traits in Louisiana Iris hybrids. Evolution 61:2308-2319.

Bradshaw, H. D., and D. W. Schemske. 2003. Allele substitution at a flower colour locus

Bradshaw, H. D., S. M. Wilbert, K. G. Otto, and D. W. Schemske. 1995. Genetic mapping of floral traits associated with reproductive isolation in monkeyflowers (Mimulus). Nature

Broman, K. W., H. Wu, Ś. Sen, and G. A. Churchill. 2003. R/qtl: QTL mapping in experimental crosses. Bioinformatics 19:889-890.

Brothers, A. N., J. G. Barb, E. S. Ballerini, D. W. Drury, S. J. Knapp, and M. L. Arnold. 2013. Genetic architecture of floral traits in Iris hexagona and Iris fulva. J. Hered. 104:853-861. variation in nectar sugar composition in two Aquilegia species (Ranunculaceae):

Chen, K. Y., B. Cong, R. Wing, J. Vrebalov, and S. D. Tanksley. 2007. Changes in regulation of a transcription factor lead to autogamy in cultivated tomatoes. Science 318:643-645. 
nutrition of pathogens. Nature 468:527-532.

Crepet, W. L. 1984. Advanced (Constant) Insect pollination mechanisms : pattern of evolution and implications vis-à-vis angiosperm diversity. Ann. Missouri Bot. Gard. 71:607-630 .

Cronk, Q., and I. Ojeda. 2008. Bird-pollinated flowers in an evolutionary and molecular context. J. Exp. Bot. 59:715-727.

Darwin, C. 1878. The effects of cross and self fertilisation in the vegetable kingdom. Project

767 Gutenberg, Urbana, IL. Retrieved February 22, 2021, from http://www.gutenberg.org/ebooks/4346

Do Carmo, M. P. 2016. Differential geometry of curves and surfaces: revised and updated second edition. Courier Dover Publications.

Fenster, C. B., W. S. Armbruster, P. Wilson, M. R. Dudash, and J. D. Thomson. 2004. Pollination syndromes and floral specialization. Annu. Rev. Ecol. Evol. Syst. 35:375-403. sequencing of rapidly evolving cpDNA regions. New Phytol. 198:579-592. 
784 Galliot, C., J. Stuurman, and C. Kuhlemeier. 2006. The genetic dissection of floral pollination 785 syndromes. Curr. Opin. Plant Biol. 9:78-82.

786 Gegear, R. J., R. Burns, and K. A. Swoboda-Bhattarai. 2017. “Hummingbird” floral traits interact synergistically to discourage visitation by bumble bee foragers. Ecology 98:489_

Gerats, A. G. M., P. de Vlaming, M. Doodeman, B. Al, and A. W. Schram. 1982. Genetic control of the conversion of dihydroflavonols into flavonols and anthocyanins in flowers of Petunia

Goodwillie, C., C. Ritland, and K. Ritland. 2006. The Genetic Basis of Floral Traits Associated with Mating System Evolution in Leptosiphon ( Polemoniaceae ): An Analysis of Quantitative Trait Loci. Evolution 60:491-504.

Grant, V. 1949. Pollination Systems as Isolating Mechanisms in Angiosperms. Evolution 3:8297.

Grimaldi, D. 1999. The Co-Radiations of Pollinating Insects and Angiosperms in the Cretaceous.

Han, Y., M. Wu, L. Cao, W. Yuan, M. Dong, X. Wang, W. Chen, and F. Shang. 2016.

Harrell, F. E. 2020. Hmisc: Harrell Miscellaneous. https://CRAN.R-project.org/package=Hmisc. 
Hermann, K., U. Klahre, J. Venail, A. Brandenburg, and C. Kuhlemeier. 2015. The genetics of reproductive organ morphology in two Petunia species with contrasting pollination syndromes. Planta 241:1241-1254.

810 Hermann, K., and C. Kuhlemeier. 2011. The genetic architecture of natural variation in flower morphology. Curr. Opin. Plant Biol. 14:60-65.

812 Hodges, S. A., and M. L. Arnold. 1995. Spurring plant diversification : are floral nectar spurs a 813 key innovation? Proc. R. Soc. B Biol. Sci. 262:343-348.

814 Hodges, S. A., M. Fulton, J. Y. Yang, and J. B. Whittall. 2004. Verne Grant and evolutionary studies of Aquilegia. New Phytol. 161:113-120.

816 Hopkins, R., and M. D. Rausher. 2011. Identification of two genes causing reinforcement in the Texas wildflower Phlox drummondii. Nature 469:411-414.

Juenger, T., J. M. Pérez-Pérez, S. Bernal, and J. L. Micol. 2005. Quantitative trait loci mapping of floral and leaf morphology traits in Arabidopsis thaliana: Evidence for modular genetic architecture. Evol. Dev. 7:259-271.

821 Katzer, A. M., C. A. Wessinger, and L. C. Hileman. 2019. Nectary size is a pollination syndrome 822 trait in Penstemon. New Phytol. 223:377-384.

823 Kishimoto, S., C. Oda-Yamamizo, and A. Ohmiya. 2018. Regulation of Carotenoid Pigmentation in Corollas of Petunia. Plant Mol. Biol. Report. 36:632-642.

Li, H., and R. Durbin. 2009. Fast and accurate short read alignment with Burrows-Wheeler transform. Bioinformatics 25:1754-1760. 
Li, L., D. J. Paolillo, M. V. Parthasarathy, E. M. DiMuzio, and D. F. Garvin. 2001. A novel gene mutation that confers abnormal patterns of $\beta$-carotene accumulation in cauliflower (Brassica oleracea var. botrytis). Plant J. 26:59-67.

Lin, I. W., D. Sosso, L. Q. Chen, K. Gase, S. G. Kim, D. Kessler, P. M. Klinkenberg, M. K. Gorder, B. H. Hou, X. Q. Qu, C. J. Carter, I. T. Baldwin, and W. B. Frommer. 2014. Nectar secretion requires sucrose phosphate synthases and the sugar transporter SWEET9. Nature 508:546-549.

Macior, L. W. 1978. Pollination Ecology of Vernal Angiosperms. Oikos 30:452-460.

Matsuba, Y., N. Sasaki, M. Tera, M. Okamura, Y. Abe, E. Okamoto, H. Nakamura, H. Funabashi, M. Takatsu, M. Saito, H. Matsuoka, K. Nagasawa, and Y. Ozekia. 2010. A novel glucosylation reaction on anthocyanins catalyzed by acyl-glucose-dependent glucosyltransferase in the petals of carnation and delphinium. Plant Cell 22:3374-3389.

Mavraganis, K. 1998. Reproductive ecology \& population biology of Aquilegia canadensis (Ranunculaceae). Queen's University, Kingston, Ontario, Canada.

Min, Y., J. I. Bunn, and E. M. Kramer. 2019. Homologs of the STYLISH gene family control nectary development in Aquilegia. New Phytol. 221:1090-1100.

Miyata, M., T. Yamamoto, T. Komori, and N. Nitta. 2007. Marker-assisted selection and evaluation of the QTL for stigma exsertion under japonica rice genetic background. Theor. Appl. Genet. 114:539-548.

Nakazato, T., L. H. Rieseberg, and T. E. Wood. 2013. The genetic basis of speciation in the Giliopsis lineage of Ipomopsis (Polemoniaceae). Heredity 111:227-237.

$\mathrm{Ng}$, J., and S. D. Smith. 2016. How to make a red flower: The combinatorial effect of pigments. AoB Plants 8:1-11. 
Noutsos, C., A. M. Perera, B. J. Nikolau, S. M. D. Seaver, and D. H. Ware. 2015. Metabolomic profiling of the nectars of Aquilegia pubescens and A. canadensis. PLoS One 10:1-13.

Ohmiya, A. 2009. Carotenoid cleavage dioxygenases and their apocarotenoid products in plants. Plant Biotechnol. 26:351-358.

Prazmo, W. 1965. Cytogenetic studies on the genus Aquilegia III: Inheritance of the traits distinguishing different complexes in the genus Aquilegia. Acta Soc. Bot. Pol. 34:403-437.

Rockman, M. V. 2012. The QTN program and the alleles that matter for evolution: All that's gold does not glitter. Evolution 66:1-17.

Roe, L. S. 1992. Status review of Aquilegia brevistyla, Lewis \& Clark National Forest. Montana

Roy, R., A. J. Schmitt, J. B. Thomas, and C. J. Carter. 2017. Review: Nectar biology: From molecules to ecosystems. Plant Sci. 262:148-164.

Rumble, J. R. (ed). 2019. CRC Handbook of Chemistry and Physics. 100th ed. CRC Press.

Sasaki, N., and T. Nakayama. 2015. Achievements and perspectives in biochemistry concerning anthocyanin modification for blue flower coloration. Plant Cell Physiol. 56:28-40. Tomancak, and A. Cardona. 2012. Fiji: An open-source platform for biological-image

872 Sheehan, H., K. Hermann, and C. Kuhlemeier. 2012. Color and scent: How single genes influence pollinator attraction. Cold Spring Harb. Symp. Quant. Biol. 77:117-133.

874 Sievers, F., A. Wilm, D. Dineen, T. J. Gibson, K. Karplus, W. Li, R. Lopez, H. McWilliam, M. 
high-quality protein multiple sequence alignments using Clustal Omega. Mol. Syst. Biol. 7:1-6.

Smith, S. D., and M. D. Rausher. 2011. Gene loss and parallel evolution contribute to species difference in flower color. Mol. Biol. Evol. 28:2799-2810.

Stracke R., Werber M., and B. Weisshaar. (2001). The R2R3-MYB gene family in Arabidopsis thaliana. Curr. Opin. Plant Biol. 4:447-456.

Stuurman, J., M. E. Hoballah, L. Broger, J. Moore, C. Basten, and C. Kuhlemeier. 2004. Dissection of floral pollination syndromes in Petunia. Genetics 168:1585-1599.

Tanaka, Y., N. Sasaki, and A. Ohmiya. 2008. Biosynthesis of plant pigments: anthocyanins, betalains and carotenoids. Plant J. 54:733-749.

Taylor, R. J. 1984. Floral Anthocyanins of Aquilegia and Their Relationship to Distribution and Pollination Biology of the Species. Bull. Torrey Bot. Club 111:462-468.

Team, R. C. 2020. R: A language and environment for statistical computing. R Foundation for Stastical Computing, Vienna, Austria.

Ureshino, K., M. Nakayama, and I. Miyajima. 2016. Contribution made by the carotenoid cleavage dioxygenase 4 gene to yellow colour fade in azalea petals. Euphytica 207:401-

Via, S., and D. J. Hawthorne. 2005. Back to the future: Genetic correlations, adaptation and speciation. Genetica 123:147-156.

Waterhouse, A. M., J. B. Procter, D. M. A. Martin, M. Clamp, and G. J. Barton. 2009. Jalview Version 2-A multiple sequence alignment editor and analysis workbench. Bioinformatics 25:1189-1191.

Wessinger, C. A., L. C. Hileman, and M. D. Rausher. 2014. Identification of major quantitative 
trait loci underlying floral pollination syndrome divergence in Penstemon. Philos. Trans. R. Soc. B Biol. Sci. 369.

901 Wessinger, C. A., and M. D. Rausher. 2013. Predictability and irreversibility of genetic changes associated with flower color evolution in Penstemon barbatus. Evolution 68:1058-1070.

Whittall, J. B., and S. A. Hodges. 2007. Pollinator shifts drive increasingly long nectar spurs in columbine flowers. Nature 447:706-709.

Wilson, P., A. D. Wolfe, W. S. Armbruster, and J. D. Thomson. 2007. Constrained lability in

Yant, L., S. Collani, J. Puzey, C. Levy, and E. M. Kramer. 2015. Molecular basis for threedimensional elaboration of the Aquilegia petal spur. Proc. R. Soc. B Biol. Sci. 282.

Yuan, Y. W., A. B. Rebocho, J. M. Sagawa, L. E. Stanley, and H. D. Bradshaw. 2016. Competition between anthocyanin and flavonol biosynthesis produces spatial pattern variation of floral pigments between Mimulus species. Proc. Natl. Acad. Sci. U. S. A.

914 Zhang, B., F. Han, H. Cui, X. Li, W. Ren, Z. Fang, L. Yang, M. Zhuang, H. Lv, Y. Liu, Y.

915 Wang, J. Song, and Y. Zhang. 2019. Insertion of a CACTA-like transposable element petals of California poppy. Plant Cell Rep. 37:1311-1323. 
bioRxiv preprint doi: https://doi.org/10.1101/2021.04.12.439277; this version posted April 13, 2021. The copyright holder for this preprint (which was not certified by peer review) is the author/funder, who has granted bioRxiv a license to display the preprint in perpetuity. It is made available under aCC-BY-NC-ND 4.0 International license.

923

924 


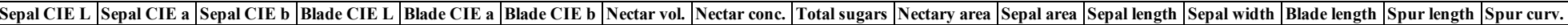

\begin{tabular}{|c|c|c|c|c|c|c|c|c|c|c|c|c|c|c|c|c|}
\hline Sepal CIE a & $-0.95 * * *$ & & & & & & & & & & & & & & & \\
\hline Sepal CIE b & $0.60^{* * * *}$ & $-0.62 * * *$ & & & & & & & & & & & & & & \\
\hline Blade CIE L & $0.14^{*}$ & \begin{tabular}{|l|}
-0.09 \\
\end{tabular} & $0.20 * * *$ & & & & & & & & & & & & & \\
\hline Blade CIE a & $\mid-0.20^{* * * *}$ & $0.17^{* * *}$ & $-0.40^{* * *}$ & $-0.28 * * *$ & & & & & & & & & & & & \\
\hline Blade CIE b & $0.19 * * *$ & $-0.15 * *$ & $0.45 * * *$ & $0.58^{* * * *}$ & $-0.73 * * *$ & & & & & & & & & & & \\
\hline Nectar vol. & 0.02 & -0.03 & 0.10 & 0.05 & $-0.15^{* *}$ & $0.12^{*}$ & & & & & & & & & & \\
\hline Nectar conc. & -0.02 & 0.05 & -0.08 & \begin{tabular}{|l|}
0.02 \\
\end{tabular} & \begin{tabular}{|l|}
0.06 \\
\end{tabular} & 0.05 & $-0.8 * * *$ & & & & & & & & & \\
\hline Total sugars & \begin{tabular}{|l|}
0.07 \\
\end{tabular} & -0.04 & 0.10 & $0.13^{*}$ & $-0.22 * * *$ & $0.29 * * *$ & $0.79 * * *$ & $-0.31 * * *$ & & & & & & & & \\
\hline Nectary area & 0.03 & $\mid-0.06$ & $0.25 * * *$ & $0.22 * * *$ & $-0.36^{* * *}$ & $0.31 * * *$ & $0.62 * * *$ & $-0.48^{* * *}$ & $0.49 * * *$ & & & & & & & \\
\hline Sepal area & $-0.16^{* *}$ & $0.16^{* * *}$ & $0.16^{* *}$ & 0.10 & $\mid-0.32 * * *$ & $0.26^{* * *}$ & $0.58 * * *$ & $-0.45^{* * *}$ & $0.47 * * *$ & $0.73 * * *$ & & & & & & \\
\hline Sepal length & $-0.17^{* *}$ & $0.16^{* *}$ & 0.03 & -0.04 & $-0.15^{* *}$ & 0.03 & $0.55^{* * * *}$ & $-0.44 * * *$ & $0.42^{* * * *}$ & $0.64 * * *$ & $0.87 * * *$ & & & & & \\
\hline Sepal width & $-0.25 * * *$ & $0.25 * * *$ & $0.18^{* *}$ & $0.18^{* * * *}$ & $-0.39 * * *$ & $0.39^{* * * *}$ & $0.45^{* * *}$ & $-0.33 * * *$ & $0.38^{* * * *}$ & $0.65 * * *$ & $0.92 * * *$ & $0.67 * * *$ & & & & \\
\hline Blade length & $\mid-0.21 * * *$ & $0.18^{* * * *}$ & $-0.38^{* * * *}$ & $-0.40^{* * * *}$ & $0.43^{* * * *}$ & $-0.55^{* * * *}$ & 0.02 & 0.02 & 0.00 & 0.04 & 0.09 & $0.34^{* * * *}$ & -0.09 & & & \\
\hline pur length & \begin{tabular}{|l|}
0.05 \\
\end{tabular} & -0.01 & $0.40^{* * * *}$ & $0.40 * * *$ & $\mid-0.47 * * *$ & $\mid 0.59^{* * * *}$ & $0.24 * * *$ & $-0.19^{* * *}$ & $0.22^{* * * *}$ & $0.46^{* * *}$ & $0.50^{* * *}$ & $0.23^{* * * *}$ & 0.60 **** & $-0.45 * * *$ & & \\
\hline pur curv. & -0.04 & 0.06 & $-0.14 * *$ & -0.07 & $0.13^{*}$ & -0.08 & $-0.20^{* * *}$ & $0.21 * * *$ & -0.11 & -0.34 & $-0.21 * * *$ & $-0.15^{* *}$ & $-0.20^{* * *}$ & $0.18^{* * * *}$ & $-0.37 * * *$ & \\
\hline istil length & \begin{tabular}{|l|}
0.02 \\
\end{tabular} & 0.00 & $0.42 * * *$ & $0.21 * * *$ & $-0.32 * * *$ & $0.35 * * *$ & $0.31 * * *$ & $-0.23 * * *$ & $0.25 * * *$ & $0.60^{* * *}$ & $0.54 * * *$ & $0.36^{* * *}$ & $0.57 * * *$ & $-0.24 * * *$ & $0.71^{* * * *}$ & \\
\hline
\end{tabular}

Table 1. Pairwise trait correlations. Cells are shaded based on absolute value of $\rho$ (Spearman correlation coefficient). ${ }^{*} 0.05,{ }^{* *} 0.01,{ }^{* * *} 0.00$ 
bioRxiv preprint doi: https://doi.org/10.1101/2021.04.12.439277; this version posted April 13, 2021. The copyright holder for this preprint (which was not certified by peer review) is the author/funder, who has granted bjoRxiv a license to display the preprint in perpetuity. It is made

\begin{tabular}{|c|c|c|c|c|c|c|c|c|c|c|}
\hline (2) & chr & $\begin{array}{l}1.5 \text { LOD } \\
\text { int }(\mathrm{cM}) \\
\end{array}$ & $\begin{array}{c}\text { availeable } \\
\text { Position } \\
\text { (cM) }\end{array}$ & $\begin{array}{c}\text { der aCC } \\
\text { LOD at } \\
\text { peak }\end{array}$ & NC-ND & $\begin{array}{l}0 \text { Internatid } \\
\text { Total PVE }\end{array}$ & $\begin{array}{l}\text { nal license. } \\
\text { Interaction }\end{array}$ & $\begin{array}{c}\text { Additive } \\
\text { Effect }\end{array}$ & \begin{tabular}{|c|} 
Dominance \\
Deviation \\
\end{tabular} & $\begin{array}{c}\text { Degree of } \\
\text { Dominance }\end{array}$ \\
\hline \multirow{3}{*}{ Sepal CIE L } & 1 & $47.34-49$ & 48.33 & 143.4 & 76.5 & \multirow{3}{*}{87} & \multirow{3}{*}{ with chr $1,1.9 \% \mathrm{VE}$} & -8 & -6.4 & 0.80 \\
\hline & 2 & $27-30.97$ & 30 & 28.3 & 6 & & & 1.9 & 1.4 & 0.74 \\
\hline & 6 & $26.2-43$ & 33.1 & 9.4 & 1.7 & & & 1.9 & -0.6 & 0.32 \\
\hline \multirow{3}{*}{ Sepal CIE a } & 1 & $47.34-49$ & 48.33 & 116.79 & 69.81 & \multirow{3}{*}{81.7} & & 5.33 & 4.34 & 0.81 \\
\hline & 2 & $28-30.97$ & 30 & 29.54 & 8.93 & & with chr $1,5.3 \% \mathrm{VE}$ & -0.83 & -0.49 & 0.59 \\
\hline & 6 & $30-37$ & 34 & 23.14 & 6.68 & & with chr $1,1.8 \% \mathrm{VE}$ & -1.42 & 0.54 & 0.38 \\
\hline \multirow{5}{*}{ Sepal CIE b } & 1 & $47.34-49$ & 48.33 & 126.1 & 56.8 & \multirow{5}{*}{87.3} & & -4.86 & -3.94 & 0.81 \\
\hline & 2 & $27-30.97$ & 28.91 & 28.6 & 6 & & with chr $1,1.9 \% \mathrm{VE}$ & -2.56 & -1.2 & 0.47 \\
\hline & 3 & $28.41-29.74$ & 29.14 & 36.9 & 8.2 & & & -2.22 & -1.52 & 0.68 \\
\hline & 4 & $58-62$ & 59 & 30.2 & 6.4 & & with chr $1,2 \% \mathrm{VE}$ & -2.31 & -1.17 & 0.51 \\
\hline & 6 & $26.22-44$ & 32 & 6.8 & 1.2 & & & -0.86 & -0.56 & 0.65 \\
\hline \multirow{5}{*}{ Blade CIE L } & 1 & $20-31.78$ & 29.22 & 4.9 & 3.1 & & & -0.34 & -0.26 & 0.76 \\
\hline & 2 & $38.13-55$ & 43 & 6.7 & 4.3 & & & 0.44 & 0.52 & 1.18 \\
\hline & 3 & $24.67-38$ & 29 & 9.1 & 5.9 & 55.3 & None & -0.18 & -0.79 & 4.39 \\
\hline & 5 & $24.03-32$ & 29 & 37.6 & 30 & & & -1.63 & 0.12 & 0.07 \\
\hline & 7 & $17-25.87$ & 22 & 13.3 & 8.9 & & & -0.68 & -0.43 & 0.63 \\
\hline & 1 & $41.18-52$ & 47 & 8.4 & 7.2 & & & 0.7 & -0.16 & 0.23 \\
\hline Blade CIE a & 4 & $21-42.16$ & 29.69 & 7.34 & 6.2 & 414 & None & 0.55 & -0.02 & 0.04 \\
\hline Blade CIE a & 5 & $28-35.97$ & 31 & 16.6 & 14.9 & 41.4 & None & 1.02 & 0.07 & 0.07 \\
\hline & 7 & $19-34.87$ & 30.32 & 5.8 & 4.8 & & & 0.48 & 0.12 & 0.25 \\
\hline & 1 & $40.19-48$ & 43.55 & 27.8 & 14 & & & -3.83 & 0.42 & 0.11 \\
\hline Blade CIE b & 3 & 25.34-30.33 & 26 & 28.9 & 14.7 & 697 & None & -3.55 & 0.1 & 0.03 \\
\hline Drade C o & 5 & 31-35.97 & 32 & 37.9 & 20.6 & 09.1 & None & -4.85 & 0.02 & 0.00 \\
\hline & 6 & $32-43$ & 38 & 6.3 & 2.7 & & & -1.55 & -0.14 & 0.09 \\
\hline & 3 & $20.25-34$ & 25.62 & 10.5 & 6.3 & & & -5.01 & 1.26 & 0.25 \\
\hline nectar volume & 4 & $32-54$ & 49.42 & 6.01 & 3.51 & 63.3 & None & 3.5 & 0.6 & 0.17 \\
\hline mectal voiume & 7 & $9.36-34.70$ & 30 & 8.5 & 5.04 & 05.5 & TNOHE & -4.45 & -0.39 & 0.09 \\
\hline & & location & covariate & & 35 & & & & & \\
\hline & 2 & $0-24.50$ & 6 & 4.3 & 1.8 & & & 3.3 & 1.2 & 0.36 \\
\hline concentration & 7 & 23.31-39 & 33.61 & 7.5 & 3.3 & 72.7 & None & 3.5 & -0.9 & 0.26 \\
\hline & & location & covariate & & 51.2 & & & & & \\
\hline & 2 & $5.31-21.12$ & 10.31 & 6.7 & 8.1 & & & 0.19 & 0.49 & 2.58 \\
\hline total sugars & 3 & $20.26-34$ & 25.35 & 5.4 & 6.5 & 24.2 & None & -0.28 & 0.06 & 0.21 \\
\hline & & location & covariate & & 5.8 & & & & & \\
\hline & 2 & $7.0-41.0$ & 20.19 & 4.2 & 3.8 & & & -0.001 & 0.001 & 1.00 \\
\hline & 3 & $17-33$ & 26.65 & 4.7 & 4.2 & & None & -0.002 & 0.001 & 0.50 \\
\hline nectary area & 4 & $38-53$ & 48.88 & 5.6 & 5.3 & 43.1 & None & 0.002 & -0.0003 & 0.15 \\
\hline & 7 & 19-26.99 & 22 & 21.7 & 22.1 & & & -0.004 & -0.0006 & 0.15 \\
\hline & 2 & 18.31-30.97 & 26.04 & 6.4 & 5.44 & & & -0.06 & 0.1 & 1.67 \\
\hline Sepal area & 3 & $8.17-28$ & 13 & 10.4 & 9.05 & 38.7 & None & -0.11 & 0.1 & 0.91 \\
\hline sepal area & 6 & $18-48$ & 43 & 8.1 & 6.94 & 38.1 & None & -0.11 & -0.01 & 0.09 \\
\hline & 7 & 18-31.69 & 20.18 & 8.3 & 7.14 & & & -0.12 & 0.04 & 0.33 \\
\hline & 2 & $18.31-30.97$ & 26.04 & 4.99 & 5.3 & & & -0.07 & 0.08 & 1.14 \\
\hline Senal length & 3 & $6.50-29$ & 10 & 4.6 & 4.9 & 226 & None & -0.04 & 0.11 & 2.75 \\
\hline Sepal Iengu & 5 & $39-54$ & 45.23 & 4.99 & 5.3 & 22.0 & & 0.1 & 0.02 & 0.20 \\
\hline & 6 & \begin{tabular}{|l|}
$0-49.53$ \\
\end{tabular} & 39 & 3.72 & 3.9 & & & -0.09 & 0.02 & 0.22 \\
\hline & 2 & $19.11-30.97$ & 26.04 & 4.45 & 3.03 & & & -0.02 & 0.03 & 1.50 \\
\hline Sepal width & 3 & $12.39-28$ & 17.6 & 19.67 & 14.89 & 50.1 & None & -0.08 & 0.02 & 0.25 \\
\hline sepal wiul & 6 & $36-50$ & 48.03 & 9.54 & 6.74 & & & -0.05 & -0.002 & 0.04 \\
\hline & 7 & 19.92-31.69 & 25 & 13.31 & 9.64 & & & -0.06 & 0.02 & 0.33 \\
\hline & 1 & $38.28-42.82$ & 41.18 & 16 & 8.9 & & & 0.05 & 0.02 & 0.40 \\
\hline & 3 & $24.05-61.12$ & 34.22 & 3.6 & 1.8 & & & 0.02 & 0.02 & 1.00 \\
\hline Blade lenoth & 4 & $24-34.50$ & 28.52 & 9.2 & 4.9 & 624 & None & 0.04 & -0.004 & 0.10 \\
\hline & 5 & $29.66-32.43$ & 31 & 31.8 & 19.8 & 02.4 & & 0.08 & 0.02 & 0.25 \\
\hline & 6 & $28.08-45.44$ & 34 & 9.8 & 5.2 & & & 0.04 & 0.01 & 0.25 \\
\hline & 7 & \begin{tabular}{|c|}
$9.0-15.0$ \\
\end{tabular} & 12 & 19.5 & 11.1 & & & 0.05 & 0.01 & 0.20 \\
\hline & 1 & $31-52.16$ & 48 & 9.2 & 4.6 & & & -0.09 & 0.02 & 0.22 \\
\hline & 2 & $18.31-35$ & 19.27 & 6.5 & 3.2 & & & -0.08 & 0.0004 & 0.01 \\
\hline Spur length & 3 & $27-43.94$ & 32.03 & 13.4 & 7 & 64.1 & None & -0.1 & 0.03 & 0.30 \\
\hline Spur length & 5 & $27.47-35.97$ & 32 & 14.4 & 7.5 & 04.1 & None & -0.12 & 0.05 & 0.42 \\
\hline & 6 & $14-40$ & 25 & 9.9 & 5 & & & -0.09 & 0.0004 & 0.00 \\
\hline & 7 & $20-25.87$ & 24.63 & 28 & 16.1 & & & -0.15 & 0.05 & 0.33 \\
\hline & 2 & $5-20.91$ & 13.09 & 3.9 & 2.5 & & & -0.11 & 0.19 & 1.73 \\
\hline $\begin{array}{l}\text { Spur } \\
\text { curvature }\end{array}$ & 4 & $22-36.59$ & 28.52 & 7.2 & 4.7 & 52.9 & None & -0.27 & -0.04 & 0.15 \\
\hline & 7 & $34.70-36.86$ & 36 & 52.1 & 46.5 & & & 0.87 & 0.16 & 0.18 \\
\hline & 1 & $25.63-39.62$ & 32.88 & 14.1 & 5.3 & & & -0.08 & -0.03 & 0.38 \\
\hline & 2 & $18.31-24.50$ & 22 & 24.6 & 9.9 & & & -0.15 & -0.01 & 0.07 \\
\hline pistil length & 3 & $8.17-32$ & 11.34 & 5.6 & 2 & 74.4 & None & -0.05 & 0.04 & 0.80 \\
\hline & 6 & $35-41.83$ & 37.49 & 9.7 & 3.5 & & & -0.08 & 0.05 & 0.63 \\
\hline & 7 & $31.28-33.33$ & 32.65 & 48.1 & 22.9 & & & -0.19 & -0.05 & 0.26 \\
\hline
\end{tabular}

Table 2. QTL data for all traits. Abbreviations: chr, chromosome; int, interval; PVE, Percent Variance Explained. 